\title{
A Combined Modal Correlation Criterion for Structural Damage Identification with Noisy Modal Data
}

\author{
Manolis Georgioudakis $\mathbb{D}^{1}$ and Vagelis Plevris $\mathbb{D}^{2}$ \\ ${ }^{1}$ Institute of Structural Analysis and Antiseismic Research, School of Civil Engineering, National Technical University of Athens, \\ Zografou Campus, Athens 15780, Greece \\ ${ }^{2}$ Department of Civil Engineering and Energy Technology, OsloMet-Oslo Metropolitan University, Pilestredet 35, \\ Oslo 0166, Norway \\ Correspondence should be addressed to Vagelis Plevris; vageli@oslomet.no
}

Received 22 August 2017; Revised 15 February 2018; Accepted 27 February 2018; Published 14 May 2018

Academic Editor: Dimitris Rizos

Copyright () 2018 Manolis Georgioudakis and Vagelis Plevris. This is an open access article distributed under the Creative Commons Attribution License, which permits unrestricted use, distribution, and reproduction in any medium, provided the original work is properly cited.

\begin{abstract}
Structural damage identification is a scientific field that has attracted a lot of interest in the scientific community during the recent years. There have been many studies intending to find a reliable method to identify damage in structural elements both in location and extent. Most damage identification methods are based on the changes of dynamic characteristics and static responses, but the incompleteness of the test data is a great obstacle for both. In this paper, a structural damage identification method based on the finite element model updating is proposed, in order to provide the location and the extent of structural damage using incomplete modal data of a damaged structure. The structural damage identification problem is treated as an unconstrained optimization problem which is solved using the differential evolution search algorithm. The objective function used in the optimization process is based on a combination of two modal correlation criteria, providing a measure of consistency and correlation between estimations of mode shape vectors. The performance and robustness of the proposed approach are evaluated with two numerical examples: a simply supported concrete beam and a concrete frame under several damage scenarios. The obtained results exhibit high efficiency of the proposed approach for accurately identifying the location and extent of structural damage.
\end{abstract}

\section{Introduction}

Structural damage identification has drawn increasing academic interest, as witnessed by the significant number of relevant journal and conference papers, during the recent years $[1-5]$. The necessity of detecting and repairing structural damage at its early stage has become imperative, and considerable effort has been devoted to developing nondestructive testing and evaluation (NDT\&E) techniques [6]. Among the NDT\&E techniques that have received significant attention in the computational mechanics field are those based on vibration signature analysis in order to obtain global information about the condition or state of health of the structural models using measured dynamic data. Such techniques use vibration characteristics of the structures including frequency response functions, natural frequencies, mode shapes, modal curvatures, and modal flexibilities to identify the occurrence of the structural damage.

Generally speaking, the existing methods of damage identification techniques based on modal testing can be classified into two major categories: direct and inverse methods. The direct methods utilize the change in modal measurement to instantly detect structural damage without the need of iterative computational procedures. In contrast, the second category of damage identification techniques poses the whole process as an inverse problem, in which the structural damage is identified by optimizing the correlation between the theoretical and the experimental modal parametric change. The level of correlation between the measured and the predicted modal characteristics is quantified using the modal correlation criteria that form 
useful mathematical tools identifying also the location and the extent of structural damage.

A class of damage identification methods belonging to the second category is based on the modification of structural model properties such as mass, stiffness, and damping to reproduce as closely as possible the measured static and dynamic responses corresponding to the experimental data [4]. These methods update the physical parameters of a finite element model of the structure by minimizing an objective function expressing the difference between finite element predicted and experimentally identified structural dynamic properties that are sensitive to damage such as natural frequencies and natural mode shapes. A thorough study of the finite element model updating approach was conducted by Friswell and Mottershead [7, 8]. Many successful applications of damage identification for detecting multiple structural damages based on the latter approach have been reported in the literature, implementing modern optimization techniques such as the evolutionary algorithms, among others [9-14].

In the present study, a structural damage identification method based on the finite element model updating is proposed, in order to provide the location and the extent of structural damage using incomplete modal data of a damaged structure. The problem of damage identification in structures is treated as an unconstrained single-objective optimization problem. The objective function of the problem to be minimized utilizes two modal correlation criteria, namely, the modal flexibility assurance criterion (MACFLEX) and the modified total modal assurance criterion (MTMAC). The combined criterion showed a superior performance in comparison to the performance of the individual criteria [15]. The performance and robustness of the proposed approach are evaluated with two numerical examples: a simply supported concrete beam and a concrete frame in several damage scenarios. The obtained results exhibit the high efficiency of the proposed approach for accurately identifying the location and extent of structural damage.

\section{Structural Damage Identification}

The problem of damage identification can be classified into four levels [16]: (A) detection, (B) localization, (C) quantification, and (D) prediction of future damage (damage prognosis). At the level of damage detection (Level A), the existence of damage can be detected, while its location and severity remain unknown. Information about location of the damage can be provided by localization techniques (Level B). At the damage quantification level (Level C), both the location and severity of damage are estimated. Finally, at the prediction level (Level D), the remaining life of the structure is estimated based on the (identified) current damage state and future loads as well as predicted damage propagation. This study reaches the third level of damage identification, which means it investigates the ability to detect, localize, and estimate the severity of damage in structures.
2.1. Damage Identification Model. It is proven that changes in the vibration characteristics of a structure are related to damage occurrence. Specifically, changes in the modal parameters, for example, natural frequencies and mode shapes, can provide an accurate indication of the presence of damage in a structure. Since modal parameters are dependent on the physical properties of the structure, that is, stiffness and mass, the finite element method (FEM) can be used as a tool for locating and quantifying damaged elements in a structure through an updating procedure of modal parameters, even for large-scale structures.

Mathematically speaking, structural damage affects the stiffness and physically the dynamic properties of the structure, such as natural frequencies and mode shapes [17]. It can be assumed that the global mass matrix remains the same in both the undamaged and the damaged state of the structure. This assumption can be considered quite accurate for the majority of practical applications. The eigenvalue problem of a structure with $n$ active degrees of freedom (DOFs) can be written as follows:

$$
\left(\mathbf{K}-\omega_{(i)}^{2} \mathbf{M}\right)\left\{\boldsymbol{\varphi}^{(i)}\right\}=0, \quad i=\{1,2, \ldots, m\},
$$

where $\mathbf{K}$ is the global stiffness matrix of the structure $([n \times n]), \mathbf{M}$ is the global mass matrix $([n \times n]),\left\{\boldsymbol{\varphi}^{(i)}\right\}$ is the $i$-th natural mode vector of the structure $([n \times 1])$ corresponding to the $\omega_{(i)}$ natural frequency, and $m$ is the total number of natural modes to be obtained $(m \leq n)$.

For a damaged structure, (1) takes the following form, respectively:

$$
\left(\mathbf{K}_{\mathrm{D}}-\omega_{\mathrm{D}(i)}^{2} \mathbf{M}\right)\left\{\boldsymbol{\varphi}_{\mathrm{D}}^{(i)}\right\}=0, \quad i=\{1,2, \ldots, m\},
$$

where $\mathbf{K}_{\mathrm{D}}$ is the global stiffness matrix of the damaged structure $([n \times n])$ and $\left\{\varphi_{\mathrm{D}}^{(i)}\right\}$ is the $i$-th natural mode vector of the damaged structure $([n \times 1])$ corresponding to the $\omega_{\mathrm{D}(i)}$ natural frequency. Equation (2) forms the basis of the damage identification method used in the present study.

2.2. Modal Correlation Criteria. We consider two structures $\mathrm{A}$ and $\mathrm{B}$ with $n$ active DOFs each, with eigenvalues $\lambda_{\mathrm{A}(i)}=$ $\omega_{\mathrm{A}(i)}^{2}$ and $\lambda_{\mathrm{B}(i)}=\omega_{\mathrm{B}(i)}^{2}$ and natural frequencies $\omega_{\mathrm{A}(i)}$ and $\omega_{\mathrm{B}(i)},(i=1,2, \ldots, m)$, where $m$ is the total number of natural modes obtained $(m \leq n)$. The corresponding mode shape vectors are $\left\{\varphi^{(i)}\right\}$ and $\left\{\psi^{(i)}\right\}([n \times 1]$ each $)$ for structures $\mathrm{A}$ and $\mathrm{B}$, respectively. In order to compare two sets of values for the two structures, the use of modal correlation criteria is imperative, providing a measure of consistency and correlation between estimations of mode shape vectors.

2.2.1. The Modal Assurance Criterion (MAC). The modal assurance criterion (MAC) $[18,19]$ is one of the most popular tools for the quantitative comparison of mode shape vectors. The purpose of this criterion is to indicate the correlation between two sets of natural modes. Considering two mode shape vectors $\left\{\boldsymbol{\varphi}^{(i)}\right\}([n \times 1])$ and $\left\{\boldsymbol{\psi}^{(j)}\right\}([n \times 1])$, for structures $\mathrm{A}$ and $\mathrm{B}$, respectively, the element $\mathrm{MAC}_{i j}$ of the MAC matrix $([m \times m])$ is given by 


$$
\begin{array}{r}
\operatorname{MAC}_{i j}=\frac{\left(\sum_{k=1}^{n} \varphi_{k}^{(i)} \psi_{k}^{(j)}\right)^{2}}{\sum_{k=1}^{n}\left[\left(\varphi_{k}^{(i)}\right)^{2}\right] \cdot \sum_{k=1}^{n}\left[\left(\psi_{k}^{(j)}\right)^{2}\right]}, \\
i, j=\{1,2, \ldots, m\} .
\end{array}
$$

$\mathrm{MAC}_{i j}$ takes values from zero, representing no consistent correspondence, to one, representing a consistent correspondence between the two mode shape vectors under consideration. In this manner, if the mode shape vectors $\left\{\boldsymbol{\varphi}^{(i)}\right\}$ and $\left\{\boldsymbol{\psi}^{(j)}\right\}$ truly exhibit a consistent relationship, the modal assurance criterion element $\mathrm{MAC}_{i j}$ approaches unity. By calculating $\mathrm{MAC}_{i j}$ for all $i, j=\{1,2, \ldots, m\}$, we obtain the MAC matrix. In addition, we define MÂC $([1 \times m])$ as the vector holding the diagonal terms of MAC matrix which can be easily calculated by setting $i=j$ in (3) [15].

\subsubsection{The Modified Total Modal Assurance Criterion (MTMAC).} One limitation of the MAC criterion is that it takes into account only the eigenvectors (mode shapes) and not the eigenvalues of the structures. This means that, in the case of uniform-like damage, the MAC criterion will not be able to detect almost any change; as in this case, the structure becomes more flexible (i.e., the eigenperiod increases), but there is no significant difference in the eigenvectors which remain almost unchanged. The natural frequencies provide global information of the structure, and they can be accurately identified through dynamic measurements.

The modified total modal assurance criterion (MTMAC) [20] is based on the MAC criterion but it also takes the corresponding eigenvalues into account. The MTMAC vector MT $\widehat{M A C}([1 \times m])$ is defined as follows:

$$
\begin{array}{r}
\operatorname{MTAMAC}_{i}=\frac{\mathrm{MAC}_{i}}{1+\left|\left(\omega_{\mathrm{A}(i)}^{2}-\omega_{\mathrm{B}(i)}^{2}\right) /\left(\omega_{\mathrm{A}(i)}^{2}+\omega_{\mathrm{B}(i)}^{2}\right)\right|}, \\
i=\{1,2, \ldots, m\},
\end{array}
$$

where $\mathrm{MAC}_{i}$ is the $i$-th component of the $\mathbf{M A} \mathbf{C}$ vector. It should be noted that the MTMAC can be easily defined also as a matrix $([m \times m])$, whose diagonal is again the MT $\widehat{M} \mathbf{A C}$ vector, as was the case with MAC. MT $\widehat{M A C}$ is a row vector with as many values as the number of natural modes considered $(m)$. By multiplying the $m$ individual values of the MTAMAC vector, we obtain the MTMAC scalar value as follows:

$$
\text { MTMAC }=\prod_{i=1}^{m} \text { MTÂAC }_{i}
$$

\subsubsection{The Modal Flexibility Assurance Criterion (MACFLEX).} It is well known that damage affects the stiffness matrix of the structure and more specifically it reduces the stiffness of the individual damaged elements. In other words, a reduction in the stiffness is equivalent to an increase in the structural flexibility. In structural health monitoring [21], it is advantageous to use changes in flexibility as an indicator of damage rather than using stiffness perturbations. This is due to the following reasons [22]:

(i) The flexibility matrix is dominated by the lower modes, and so good approximations can be obtained even when only a few lower modes are employed.

(ii) The flexibility matrices are directly attainable through the modes and mode shapes determined by the system identification process.

(iii) Iterative algorithms usually converge the fastest to high eigenvalues.

(iv) In flexibility-based methods, these eigenvalues correspond to the dominant low-frequency components in structural vibrations.

Therefore, the dynamically measured flexibility matrix which is calculated from the identified modal parameters can be used as a damage identification measure [14]. The flexibility matrix $\mathbf{F}_{\mathrm{A}}([n \times n])$ for the structure $\mathrm{A}$ is given by

$$
\mathrm{F}_{\mathrm{A}}=\boldsymbol{\Phi} \cdot \Lambda_{\mathrm{A}}^{-1} \cdot \boldsymbol{\Phi}^{\mathrm{T}}
$$

where $\Phi$ is a matrix $([n \times m])$ containing all the $m$ mode shape vectors $\left\{\varphi^{(i)}\right\}([n \times 1]$ each $)$ and $\Lambda_{\mathrm{A}}$ is a diagonal ma$\operatorname{trix}([m \times m])$ which holds the eigenvalues $\lambda_{\mathrm{A}(i)}=\omega_{\mathrm{A}(i)}^{2}, \quad(i=$ $1,2, \ldots, m)$ on its diagonal. The elements of matrix $\mathbf{F}_{\mathrm{A}}$ can be also obtained separately using the following formula:

$$
\mathrm{F}_{\mathrm{A}, i j}=\sum_{k=1}^{m} \frac{1}{\omega_{(k)}^{2}} \varphi_{k}^{(i)} \varphi_{k}^{(j)} .
$$

Each column of the flexibility matrix represents the displacement pattern of a structure associated with a unit force applied to the associated degree of freedom. As shown in (7), as the value of frequency decreases (i.e., the eigenperiod increases), the modal contribution to the flexibility matrix also increases. As a result, a good estimate of the flexibility matrix can be calculated even with a small number of the first low-frequency modes [15].

In order to compare the values of the flexibility matrix of the two structures A and B, the modal flexibility assurance criterion (MACFLEX) is applied. The $i$-th component of the MACF̂EX vector $([1 \times n])$ can be calculated as follows:

$$
\operatorname{MAC\widehat {FLEX}}_{i}=\frac{\left(\widehat{\mathbf{F}}_{\mathrm{A}}^{(i) T} \widehat{\mathbf{F}}_{\mathrm{B}}^{(i)}\right)^{2}}{\left(\widehat{\mathbf{F}}_{\mathrm{A}}^{(i) T} \widehat{\mathbf{F}}_{\mathrm{A}}^{(i)}\right)\left(\widehat{\mathbf{F}}_{\mathrm{B}}^{(i) T} \widehat{\mathbf{F}}_{\mathrm{B}}^{(i)}\right)},
$$

where $\widehat{\mathbf{F}}_{\mathrm{A}}^{(i)}$ and $\widehat{\mathbf{F}}_{\mathrm{B}}^{(i)}$ are the $i$-th column vectors $([n \times 1])$ of the flexibility matrices $F_{A}$ and $F_{B}$, for structures $A$ and $B$, respectively. MAC $\widehat{F} L E X$ is a vector with as many values as the number of columns in the flexibility matrices. Again, we could consider a full MACFLEX matrix by taking different vectors into account, instead of the $i$-th vector for both structures, but there is no point in that as again the diagonal terms of the MACFLEX matrix are the important ones. By multiplying the $n$ individual values of the MACF $\widehat{F} L \mathbf{E}$ vector, we obtain the MACFLEX scalar value as follows: 


$$
\text { MACFLEX }=\prod_{i=1}^{n} \text { MACF̂LEX }_{i}
$$

\section{Damage Identification as an Optimization Problem}

3.1. Problem Formulation. The structural damage identification problem is treated as an unconstrained optimization problem. The objective function $F$ to be minimized utilizes the two modal correlation criteria (MACFLEX and MTMAC), while the design variable $d_{i}$ describes the extent of damage of the $i$-th element of the structure. In this sense, the number of design variables is equal to the number of elements $\left(n_{\mathrm{e}}\right)$ in the structure.

In this study, the standard beam finite elements are used to represent the structures of the numerical examples. It has been assumed that no alteration of the mass occurs before and after damage, which is acceptable for most engineering applications. Therefore, the parameterization of the damage is represented by a reduction factor or damage index of the element bending stiffness. This damage index, $d_{i}$, for a damaged element $i$ represents the relative variation of the damaged element bending stiffness, $(\mathrm{EI})_{i, \mathrm{D}}$ to the initial (undamaged) bending stiffness $(\mathrm{EI})_{i}$, as follows:

$$
d_{i}=1-\frac{(\mathrm{EI})_{i, \mathrm{D}}}{(\mathrm{EI})_{i}}
$$

This definition of the damage index for each element of the structure allows estimating not only the damage extent but also the damage location since the damage identification is carried out at the individual element level. The damage index can take values between 0 (no damage) and $1(100 \%$ damage), although for numerical stability purposes, in the present study, the maximum damage has been limited to a value slightly below unity (i.e., 0.999), otherwise the structure will become a mechanism that cannot be analyzed and numerical instabilities will occur.

Taking into account all of the above, the mathematical formulation of the structural damage identification problem takes the following form:

$$
\begin{array}{lc}
\min & F\left(d_{i}\right) \\
\text { subject to } & 0<d_{i}<0.999
\end{array} \quad i=\left\{1,2, \ldots, n_{\mathrm{e}}\right\}
$$

3.2. The Objective Function. Setting up an objective function, selecting the updating parameters, and using robust optimization algorithms are three crucial steps in structural identification. They require deep physical insight and usually also trial-and-error procedures. The objective function has to reflect the deviation between the numerical prediction and the real behavior of the structure. For this reason, an objective function may be formulated in terms of the discrepancy between the finite element model and experimental quantities.

In this study, a combined objective function has been implemented, which takes into account the values of two different modal correlation criteria (MTMAC and MACFLEX) that express the relationship between the real (experimentally measured) damage and the damage which is numerically predicted by the finite element model. In particular, the objective function of the optimization problem is the following:

$$
F=\sqrt{F_{1}^{2}+F_{2}^{2}}
$$

where $F_{1}$ and $F_{2}$ are the values of the corresponding individual objective functions

$$
\begin{aligned}
& F_{1}=1-\text { MTMAC } \\
& F_{2}=1-\text { MACFLEX. }
\end{aligned}
$$

The minimum value (target value) for the objective function $F$ is zero, which is also the case for the individual objective functions $F_{1}$ and $F_{2}$.

3.3. Noise Simulation for Experimental Data. In a real-life scenario, the vibration characteristics (eigenvalues and eigenmodes) of the real damaged structure would have to be determined (measured) by experiment. In our case, for practical reasons and due to lack of appropriate experimental data, these properties are also calculated numerically using a finite element "real damage" model. This approach is acceptable for the purposes of the present study and does not cause any limitations to the proposed methodology. Thus, in the present study, the term "experimental data" has nothing to do with a real experiment but rather corresponds to simulated numerical data of the "damaged" structure. At this point, it needs to be clarified that although the real damage information is used in order to generate the "damaged" model and obtain the "experimental" data, this information is not made available to the optimization algorithm. The optimization algorithm only considers the "experimental" modal data and tries to find the damage based only on them.

Real experimental data acquired from experiments can never be perfectly accurate. During modal testing, it is usual to assume that the frequencies of vibration are accurately determined and that is in the determination of the amplitudes of the mode shapes that the experimental errors will occur. This assumption is usually valid since the frequency of shakers, even at resonance, can be quite accurately controlled. In order to simulate this phenomenon and be more compatible with real experimental values of modal parameters, the "experimental data" in the present study have been "enriched" by adding an artificial noise into them using the following equation [23]:

$$
\varphi_{i}^{(j, \exp )}=\varphi_{i}^{(j, \text { cal })} \cdot(1+\mathrm{NR} \cdot \xi),
$$

where $\varphi_{i}^{(j, \exp )}$ is the $i$-th component of the $j$-th experimental mode shape vector, $\varphi_{i}^{(j, \text { cal })}$ is the $i$-th component of the $j$-th calculated mode shape vector, NR (noise ratio) is the percentage of noise added to the calculated data (usually 0$10 \%)$, and $\xi$ is a uniformly distributed random number in the range of $(-1,1)$. 
3.4. The Differential Evolution Algorithm. Choosing the proper search algorithm for solving an optimization problem is not a straightforward procedure. In the past, a number of studies have been published where structural optimization problems have been solved using metaheuristic search algorithms and especially those based on adopting Darwinian principles of evolutionary process. These algorithms achieve efficient performance for a wide range of combinatorial optimization problems. Among the plethora of such algorithms, the differential evolution (DE) algorithm is adopted in this study to solve the structural damage identification problem of Section 3.1. DE is considered a fast and efficient metaheuristic having gained increased popularity in the engineering optimization community, with numerous applications in various research fields [24].

Differential evolution (DE) is a stochastic populationbased evolutionary algorithm for global optimization, introduced by Storn and Price [25]. It follows the standard evolutionary algorithm flow with some significant differences in the mutation and selection processes. The simplicity of $\mathrm{DE}$ algorithm is based on only three tunable parameters: the mutation factor $F_{\mathrm{m}} \in[0,2]$, the crossover probability $\mathrm{CR} \in[0,1]$, and the total number of particles (population size) NP. The fundamental idea behind DE is the use of vector differences by choosing randomly selected vectors and then taking their difference as a means to perturb the parent vector with a special kind operator and probe the search space. Several variants of DE have been proposed so far [26], but the present study is focused on the nominal approach (DE/rand/1/bin). According to this, each of the members of the population undergoes mutation and crossover. Once crossover occurs, the offspring is compared to the parent and the one with the best fitness between the two moves to the next generation (selection process).

In more detail, we consider an optimization problem with $D$ dimensions. First, all individuals $\mathbf{x}$ of the population are initialized at random positions in the search space. After initialization, each member of the population $\mathbf{x}$ undergoes mutation and a donor vector $\mathbf{v}$ is generated such as

$$
\mathbf{v}=\mathbf{a}+F_{\mathrm{m}} \cdot(\mathbf{b}-\mathbf{c}),
$$

where $\mathbf{a}, \mathbf{b}$, and $\mathbf{c}$ are three individuals from the population at random, which must be distinct from each other and from individual $\mathbf{x}(\mathbf{x} \neq \mathbf{a} \neq \mathbf{b} \neq \mathbf{c})$.

In the next step, the crossover operator is applied by generating the trial vector $\mathbf{u}$. The $i$-th component of $\mathbf{u}$ is defined either from the $i$-th component $\left(\mathrm{v}_{i}\right)$ of $\mathbf{v}$ or the $i$-th component $\left(\mathrm{x}_{i}\right)$ of $\mathbf{x}$, with probability $\mathrm{CR}$ as follows:

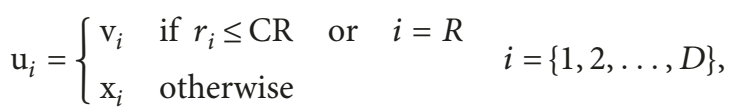

where $r_{i}$ is a random number with uniform distribution, $r_{i} \in \mathrm{U}[0,1]$, and $R$ is a random integer in $[1,2, \ldots, D]$, which ensures that, in any case, after the crossover operation at least one component is changed and thus $\mathbf{u} \neq \mathbf{x}$. The last step of the generation procedure is the implementation of the selection operator where the target vector $\mathbf{x}$ is compared to the trial vector $\mathbf{u}$. If the trial vector $\mathbf{u}$ has a better fitness value than $\mathbf{x}$, then the individual $\mathbf{x}$ is replaced in the population with the trial vector $\mathbf{u}$ as follows:

$$
\mathbf{x}^{\prime}=\left\{\begin{array}{ll}
\mathbf{u} & \text { if } f(\mathbf{u})<f(\mathbf{x}) \\
\mathbf{x} & \text { otherwise }
\end{array},\right.
$$

where $f$ is the objective function to be minimized and $\mathbf{x}^{\prime}$ is the new design vector for the next generation. If any component of the vector $\mathbf{v}$ obtained from (15) is out of the given bounds, then the specific component is reset by assigning a random value to it within the given bounds. This ensures that the final vector $\mathbf{x}^{\prime}$ from (17) will always have all values within the given range.

The optimization procedure is finished when a desirable convergence criterion has been satisfied. In the present study, the termination criterion is met when the maximum number of generations has been reached and no other convergence criterion has been used, for reasons of uniformity.

\section{Numerical Examples}

Two different structures have been investigated to show the effectiveness of the proposed methodology for identifying the location and extent of damage in structures. The first structure is a simply supported concrete beam, while the second is a concrete frame. The finite element model for both examples is based on an Euler-Bernoulli assumption of the planar elements with three degrees of freedom per node (two translational and one rotational). The mutation factor $f$ and the crossover probability CR of the DE algorithm are common in both problems with values $F_{\mathrm{m}}=0.6$ and $\mathrm{CR}=0.9$, respectively. The maximum number of generations is also common for both problems, with value 3000 . Due to the different numbers of design variables between the two problems, the population size is different, taken as $\mathrm{NP}=40$ for the beam problem and NP $=100$ for the computationally more intensive frame problem.

To check the robustness of the proposed methodology, one to four vibration modes are considered, and due to the limited number of sensors, the mode shape vector and the vertical displacements are supposed to be only read at a limited number of locations corresponding to the vertical degrees of freedom of the structures (monitored DOFs). This means that the eigenmode information (displacements) that corresponds to the experimental data is obtained for these DOFs only. The discretization of the model is an important issue that can affect the results, to some extent, but if a dense FE mesh is used, then the damage location can be approximated with enough accuracy.

A network of sensors is needed to record the eigenvectors and eigenvalues of the damaged structure. In the present study, the DOF locations correspond to the locations of the sensors, but this does not have to be the case in general. The recorded locations do not have to be the same as the DOFs of the finite element model and can be completely different, although when compared, the two systems have to be consistent with each other (comparison at the same locations). This means that, for example, the FEM mesh can be denser than the network of sensors but the comparison has to be made at the same predefined locations. 
Furthermore, in order to assess the performance of the optimization procedure, we introduce a metric which indicates how well the optimization algorithm manages to find the real damage, by means of a single scalar value. The prediction error between real damage vector $\mathbf{x}_{\text {real }}$ and the predicted damage vector $\mathbf{x}_{\text {current }}$ of the best individual of the current generation step is defined using the Euclidean norm as follows:

$$
\text { Norm }=\left\|\mathbf{x}_{\text {real }}-\mathbf{x}_{\text {current }}\right\| .
$$

The smaller the value of the Norm, the better the prediction. In case of a perfect prediction $\left(\mathbf{x}_{\text {real }}=\mathbf{x}_{\text {current }}\right)$, the Norm takes the value of zero.

4.1. Simply Supported Beam. A simply supported beam $[15,27]$ is analyzed in this section to illustrate the effectiveness of the proposed methodology and the performance of the different objective functions $\left(F_{1}, F_{2}\right.$, and $\left.F\right)$. The geometry, boundary conditions, and the finite element mesh of the beam are shown in Figure 1. The beam has a total length of $6 \mathrm{~m}$, and it is discretized by 10 equal length beam elements of rectangular cross section $b$ (width) $\times h$ (height) $=0.25 \mathrm{~m} \times 0.20 \mathrm{~m}$. The beam is considered to have Young's modulus $E$ equal to $30 \mathrm{GPa}$ and a density $\rho$ equal to $2500 \mathrm{~kg} / \mathrm{m}^{3}$. The number of monitored DOFs is 9 , and the first four eigenmodes of the undamaged structure are shown in Figure 2.

Four different damage scenarios are considered: (a) a single-element damage scenario; (b) a two-element damage scenario; (c) a three-element damage scenario; and (d) a uniform damage scenario, as shown in Figure 3.

The same optimization algorithm has been applied to all damage scenarios. For each damage scenario, the combined criterion has been used for the formulation of the objective function. For each criterion, the number of known eigenmodes varies from one to four. First, the problem is solved assuming no noise for the data, then noise is added with noise ratio $\mathrm{NR}=5 \%$ and $\mathrm{NR}=10 \%$.

\subsubsection{No Noise}

(1) Combined Criterion Results. The performance and the corresponding results of the two individual objective functions $F_{1}$ and $F_{2}$ that $F$ is based on have been already investigated by the authors, and details can be found in [15]. In light of this, in the present study, for this numerical example, we will focus on the new combined criterion only. The optimized values of the design variables for the combined objective function $F$ are shown in Figure 4, for all damage scenarios. The results are presented in bar charts, where the target damage (real damage) is always denoted in red and the other colored bars denote the damage estimation by the optimization algorithm, for different numbers of known eigenmodes.

Table 1 shows the value of the norm of (18) for each case. Norm values near zero mean perfect match between the computed and the real structural damage. In all the examined cases with no noise, the optimization algorithm manages to give good results and the real damage is

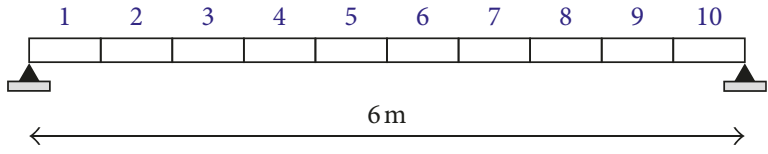

FIGURE 1: The beam structure under investigation.

estimated even with a small number of known eigenmodes. The fourth damage scenario (uniform damage) appears to be the most difficult case where a higher number of eigenmodes are needed. The estimation in the case of uniform damage is not $100 \%$ accurate even with four known eigenmodes, as shown in Figure 4(d) and the last column of Table 1.

(2) Combined Criterion versus the Individual Criteria. We investigate the performance of the combined objective function $F$ in comparison to the performance of the two individual objective functions $F_{1}$ and $F_{2}$ that have already been studied by the authors in [15]. Figure 5 shows the norm value for each of the three objective functions versus the number of generations, as the optimization problem proceeds, for the second and the third damage scenarios, with three known eigenmodes. The $x$-axis is in logarithmic scale for better clarity of the results.

The combined objective function (red line) manages to converge faster and more accurately to the solution of the problem for both damage scenarios. Due to space limitations, here, we present only the results of scenarios 2 and 3 with three known eigenmodes. But the same trend has been observed in all the cases that were examined, for all four damage scenarios and with any number of known eigenmodes $(1,2,3$, or 4$)$.

(3) Influence of the Number of Known Eigenmodes. It is interesting to investigate how the different number of known eigenmodes affects the results of the optimization procedure. It is expected that richer modal information (i.e., more known eigenmodes) should lead to more accurate results for the optimization procedure. Figure 6 presents the norm values for the combined objective function versus the number of generations for damage scenarios 2 and 3, where the different lines represent the different numbers of known eigenmodes (1 to 4 ). It is clear that the best results are obtained with four known eigenmodes and that the richer modal information leads to better results in general. This was a general observation also with other damage scenarios and also for any objective function $\left(F, F_{1}\right.$, and $\left.F_{2}\right)$.

4.1.2. Noise 5\%: Combined Criterion. In the next part of the investigation, noise is added to the experimental data, with noise ratio $\mathrm{NR}=5 \%$. In Figure 7, the optimized values of the design variables are presented for the combined objective function $F$, for all damage scenarios, for different numbers of known eigenmodes (from one to four).

As shown in Figure 7 and Table 2, when noise 5\% is considered, more eigenmodes are needed in order to achieve good results. In particular, when only one eigenmode is known, the results appear not to be so good (min 


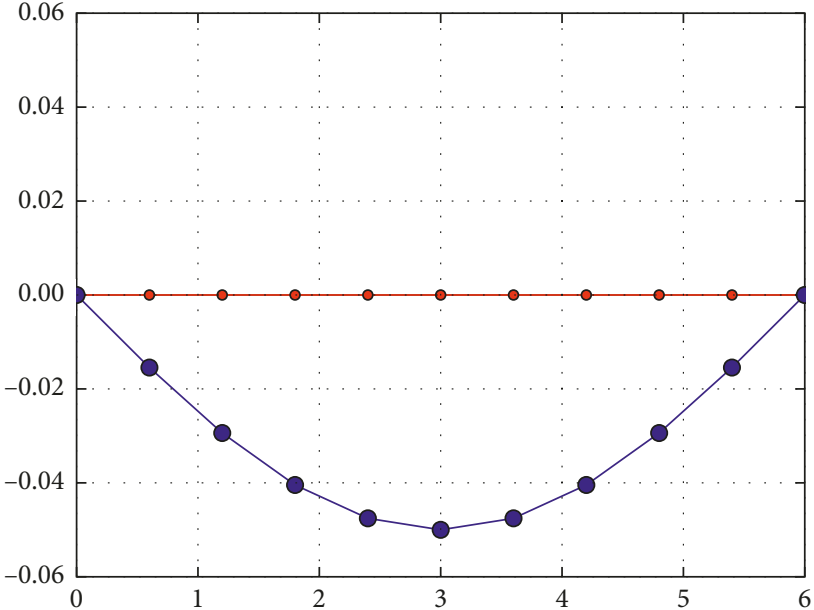

(a)

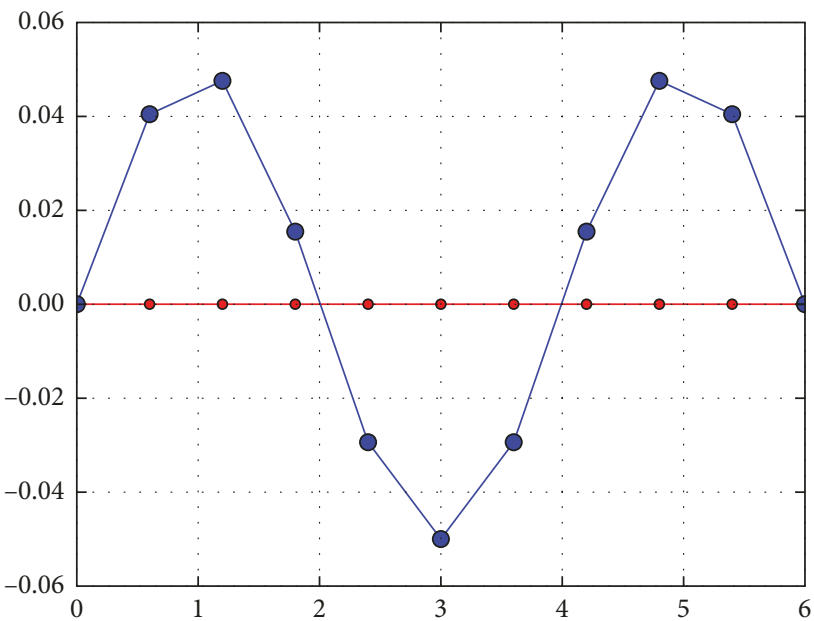

(c)

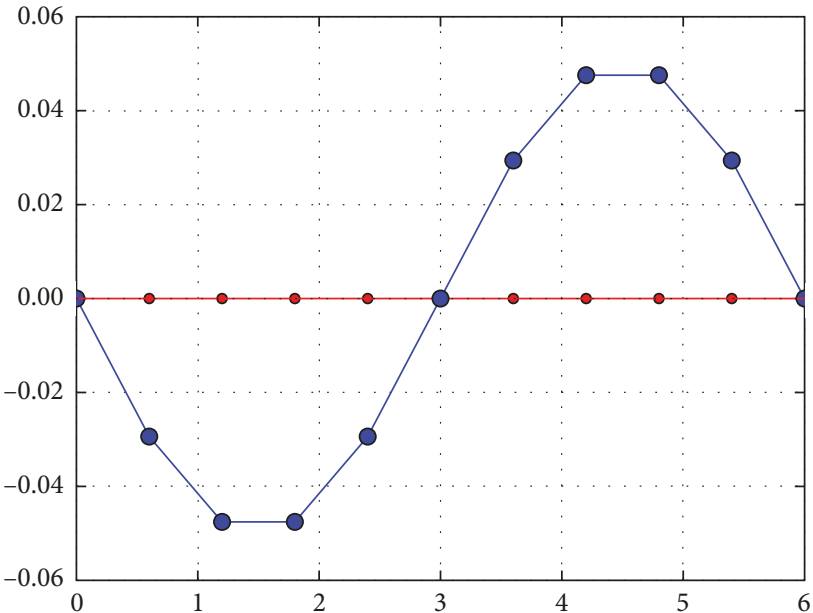

(b)

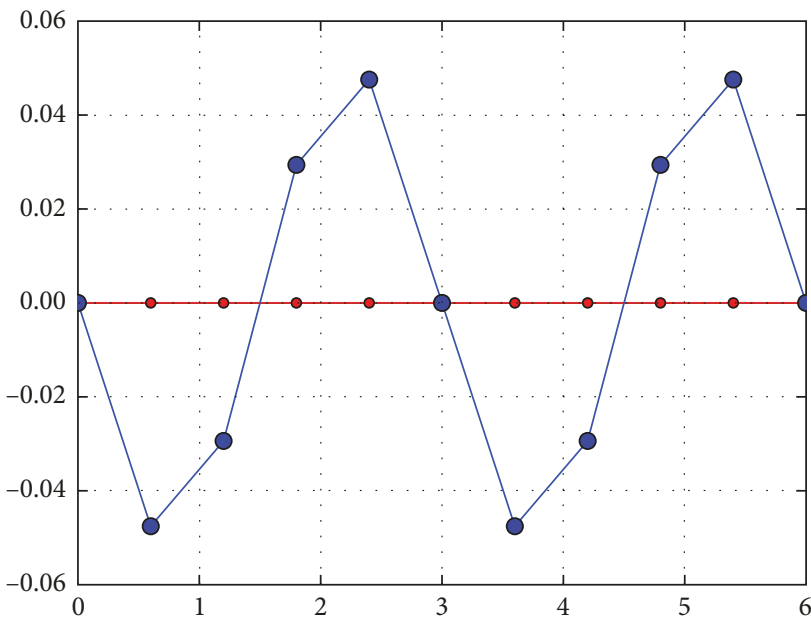

(d)

Figure 2: Beam example: first four eigenmodes of the undamaged structure. (a) Eigenmode $1\left(T_{1}=0.115 \mathrm{~s}\right),(\mathrm{b})$ eigenmode $2\left(T_{2}=0.029 \mathrm{~s}\right)$, (c) eigenmode $3\left(T_{3}=0.013 \mathrm{~s}\right)$, and $(\mathrm{d})$ eigenmode $4\left(T_{4}=0.007 \mathrm{~s}\right)$.

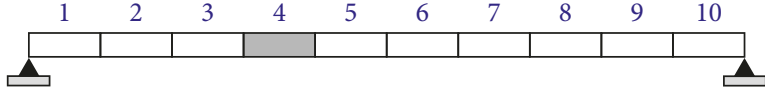

$\square 40 \%$

(a)

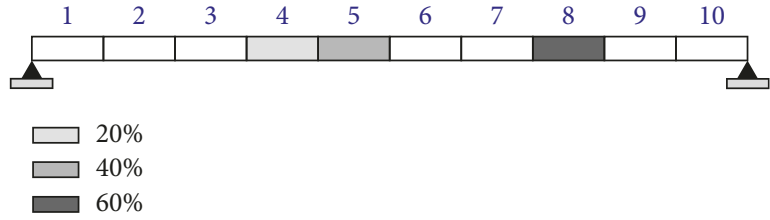

(c)

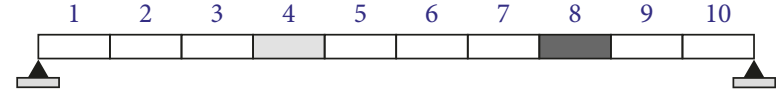

$\square 20 \%$

(b)

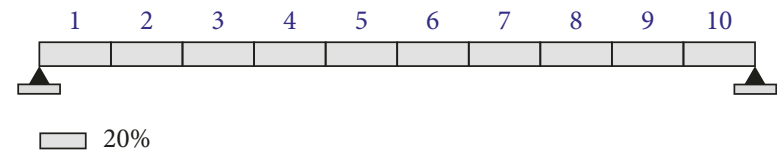

(d)

Figure 3: Beam example: the four different damage scenarios. (a) Scenario 1, (b) scenario 2, (c) scenario 3, and (d) scenario 4.

norm $=0.137$, $\max$ norm $=0.425)$, while in cases with two or more known eigenmodes, the algorithm manages to converge to good or at least acceptable results $(\min$ norm $=0$, $\max \operatorname{norm}=0.313)$.
4.1.3. Noise 10\%: Combined Criterion. Next, noise is added to the experimental data, with a higher noise ratio $\mathrm{NR}=10 \%$. In Figure 8, the optimized values of the design variables are presented for the combined objective function $F$, for all 


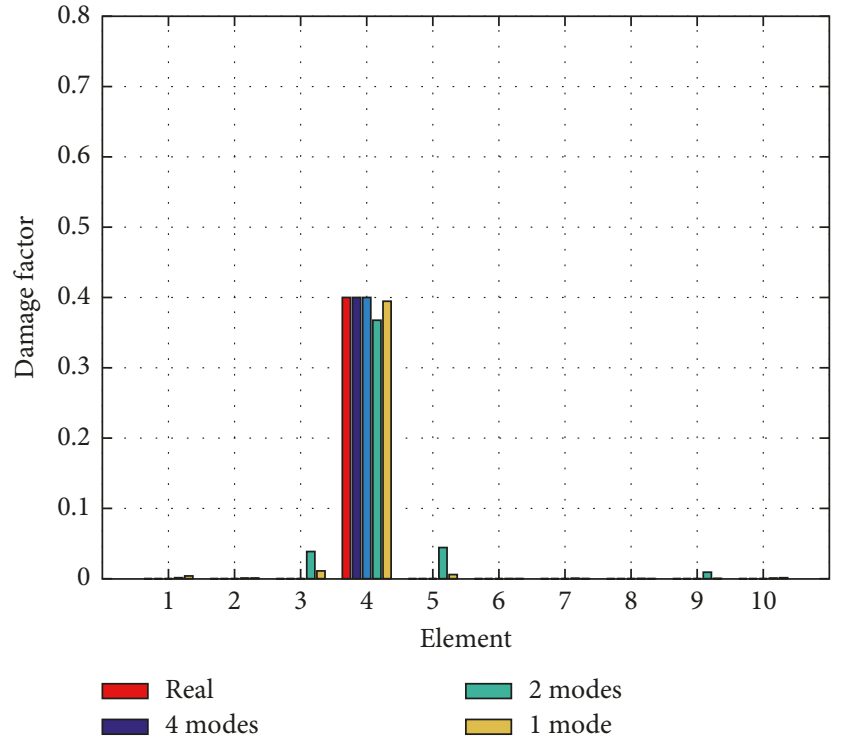

(a)

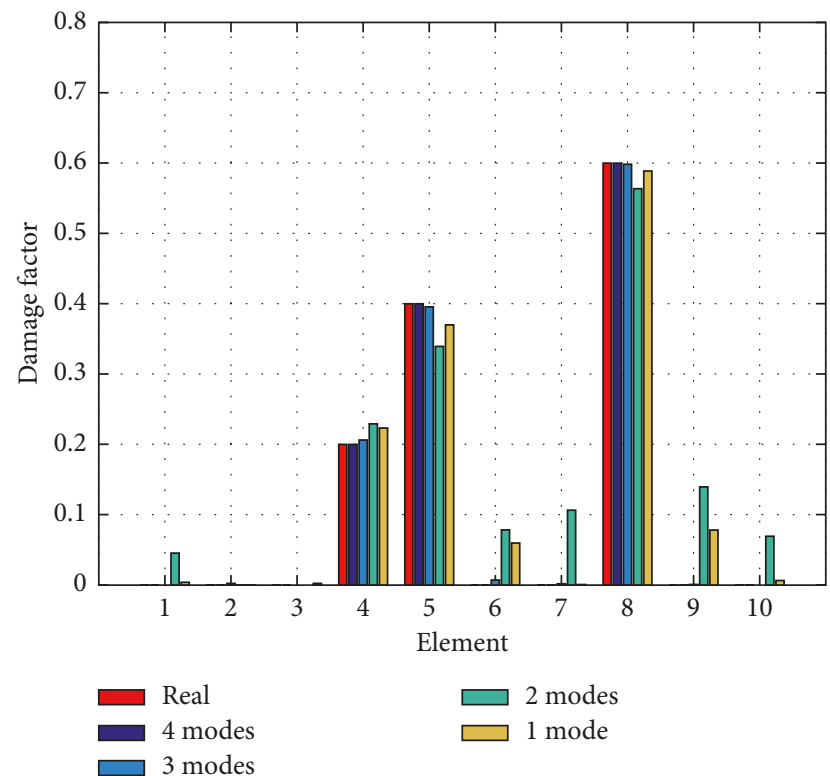

(c)

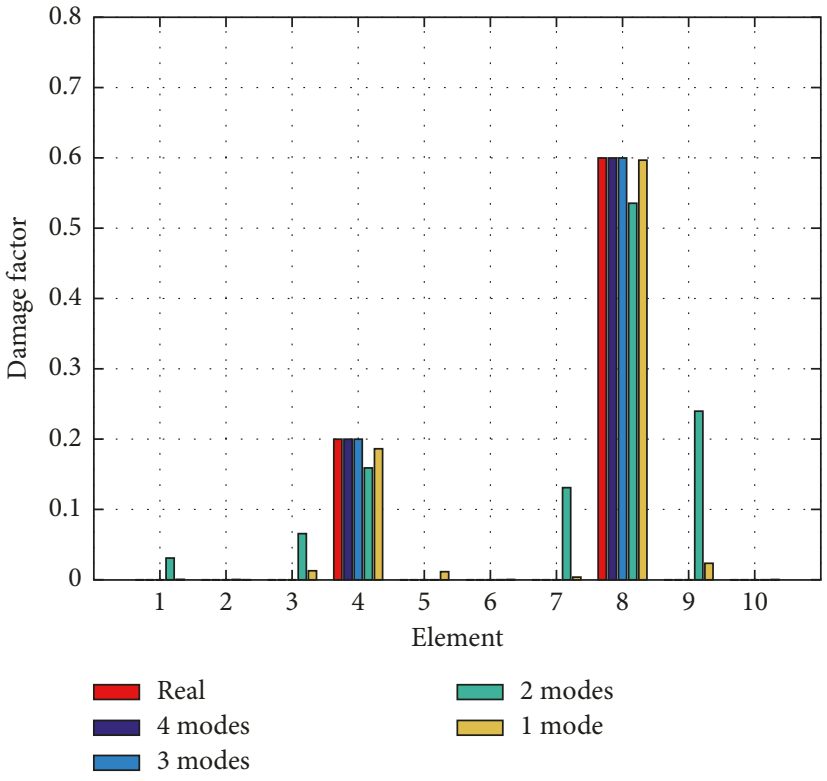

(b)

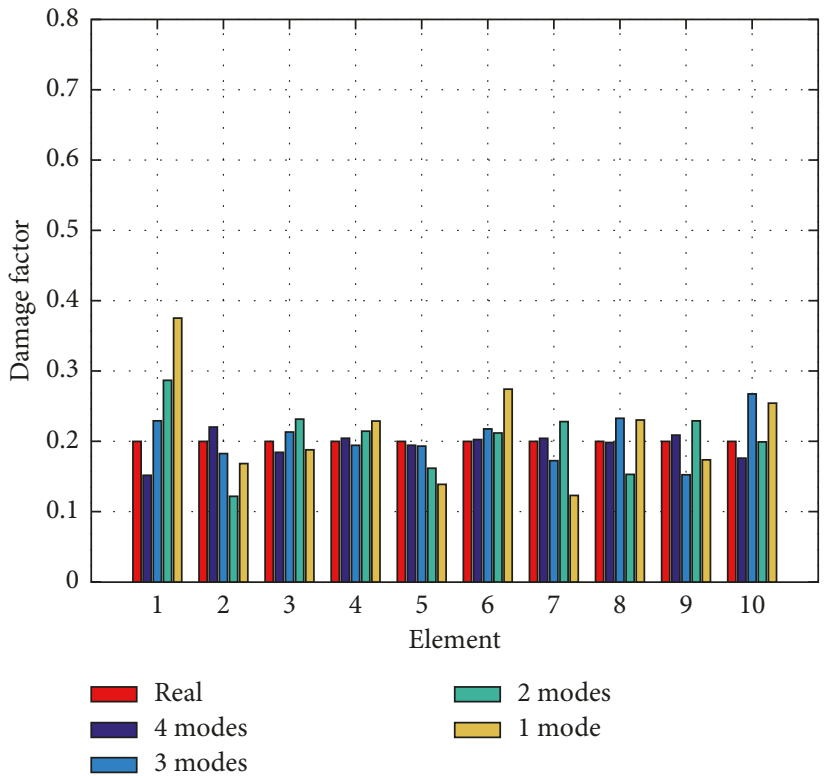

(d)

Figure 4: Beam example: damage prediction of the combined criterion for the four different damage scenarios (no noise). (a) Scenario 1, (b) scenario 2, (c) scenario 3 , and (d) scenario 4 .

TABle 1: Beam example: norm values for different damage scenarios and 1 to 4 known modes (no noise).

\begin{tabular}{lcccc}
\hline Known modes & \multicolumn{3}{c}{ Damage scenario } & 3 \\
\hline 1 mode & 1 & 2 & 0.106 & 4 \\
2 modes & 0.014 & 0.033 & 0.223 & 0.229 \\
3 modes & 0.068 & 0.293 & 0.011 & 0.143 \\
4 modes & 0.000 & 0.000 & 0.000 & 0.102 \\
\hline
\end{tabular}

damage scenarios, for different numbers of known eigenmodes (from one to four).

The case with severely distorted noisy data $(\mathrm{NR}=10 \%)$ is even more difficult, as shown in Figure 8 and Table 3, but even in this case, the optimization algorithm manages to give acceptable results and the real damage is estimated even with a small number of known eigenmodes (two or more). The case of only one known eigenmode is still problematic 


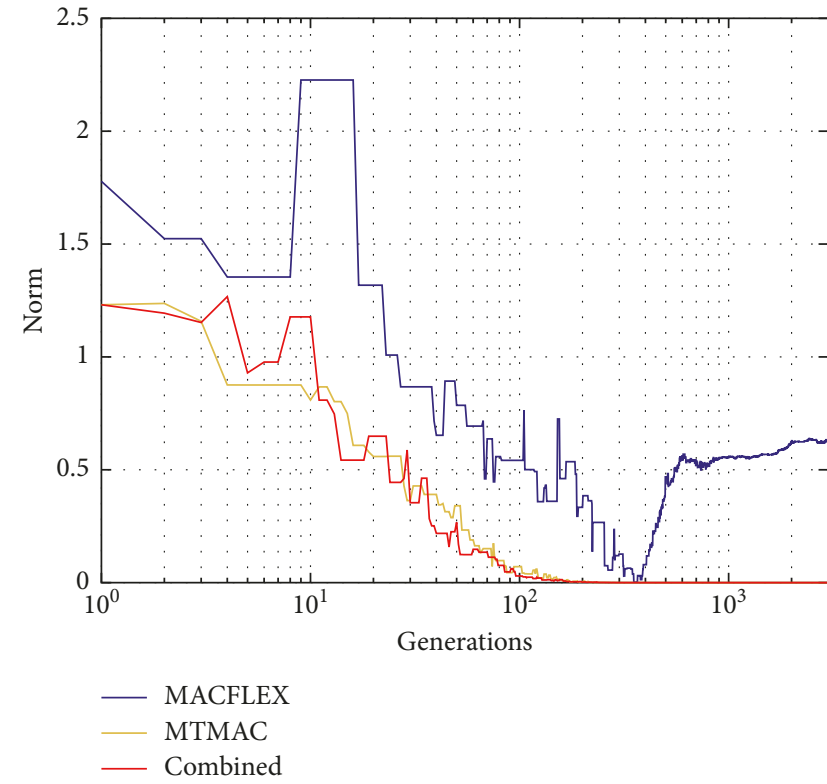

(a)

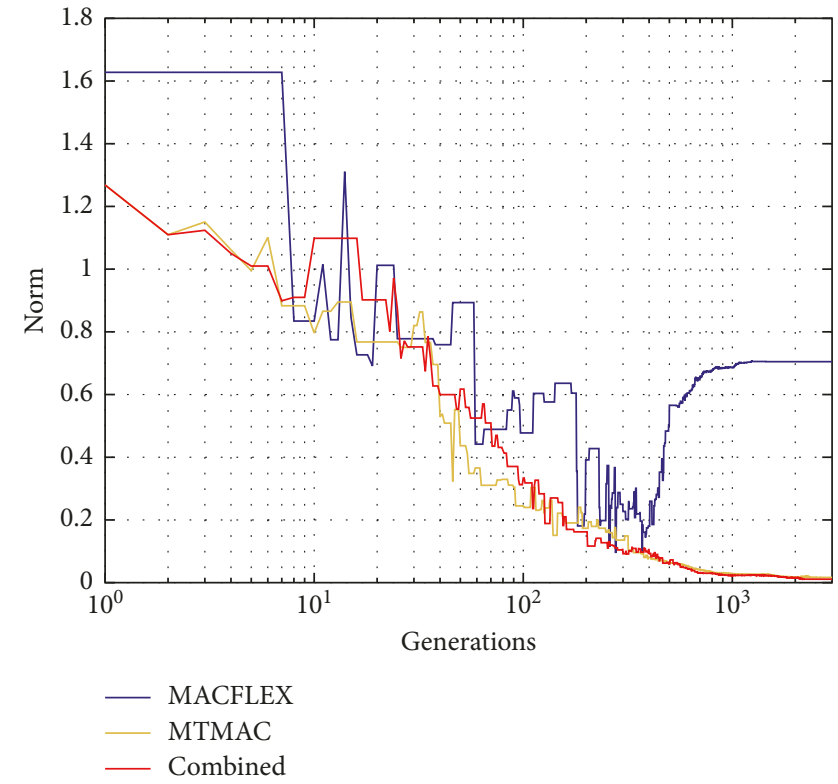

(b)

FIgURE 5: Beam example: norm values for the three objective functions versus generations, for three known eigenmodes, for damage scenarios 2 and 3 (no noise). (a) Scenario 2 and (b) scenario 3.

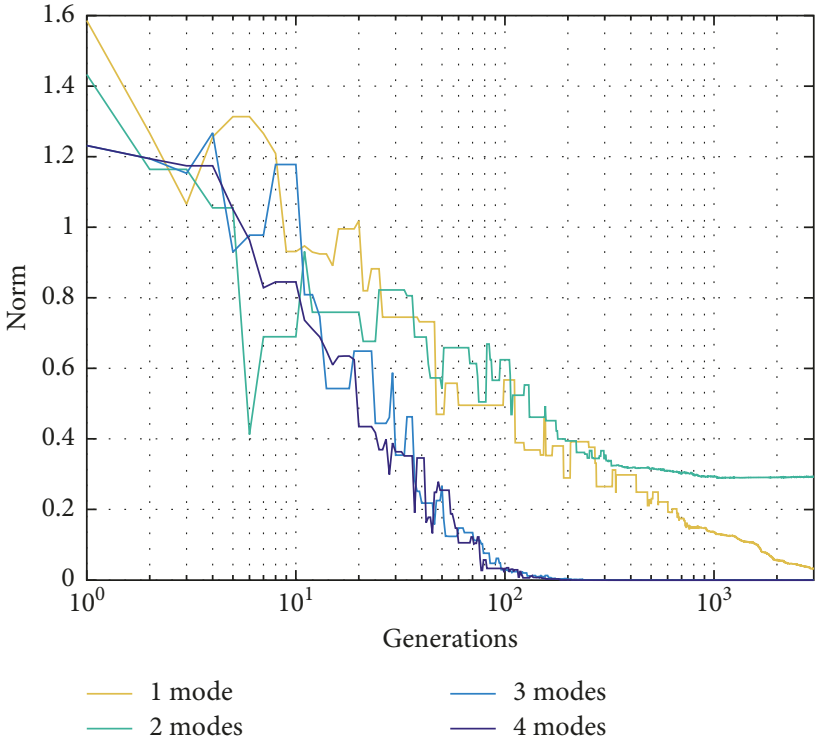

(a)

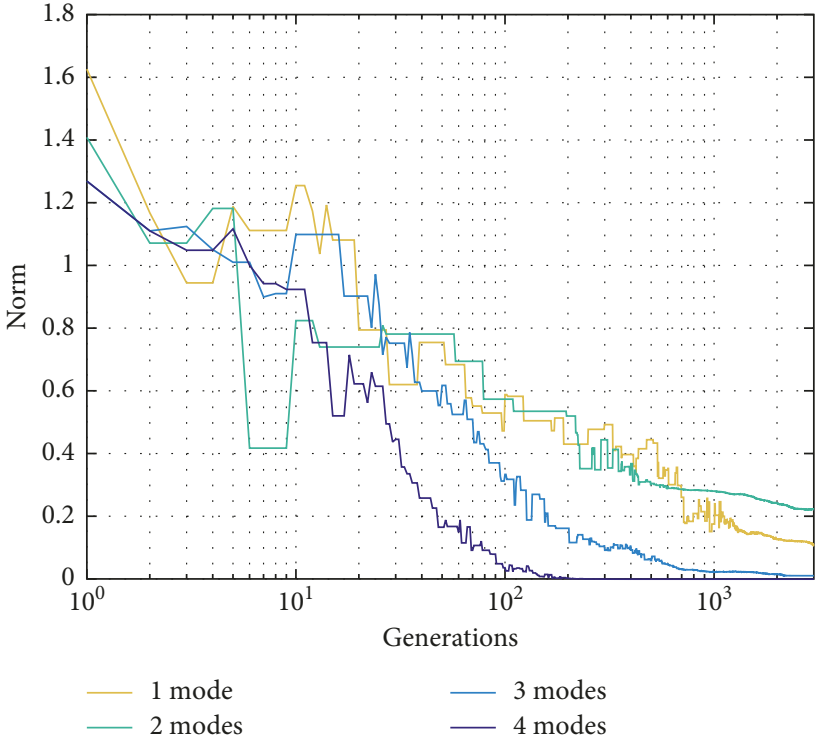

(b)

FIGURE 6: Beam example: norm values for the combined objective function, for different numbers of known eigenmodes, for damage scenarios 2 and 3 (no noise). (a) Scenario 2 and (b) scenario 3.

$(\min \operatorname{norm}=0.259, \max$ norm $=0.651)$. Again, the fourth damage scenario appears to be the most difficult one (min norm $=0.222$ for four known modes, $\max$ norm $=0.518$ for one mode).

4.2. Single-Span Concrete Frame. The second structure that is examined is a concrete frame with a span of $6 \mathrm{~m}$ and a height of $4 \mathrm{~m}$. Both supports are supposed to be fixed. The geometry and the finite element mesh of the frame are shown in Figure 9.

The frame is discretized with 28 equal length beam finite elements (12 elements for the beam and 8 elements for each column). The length of each element is $0.50 \mathrm{~m}$. The total number of nodes is 29 , while the total number of DOFs is 87 , of which 81 are active and 6 are constrained (fixed supports). The horizontal elements (beam) have a square cross section $0.25 \mathrm{~m} \times 0.25 \mathrm{~m}$, while the vertical elements (columns) have 


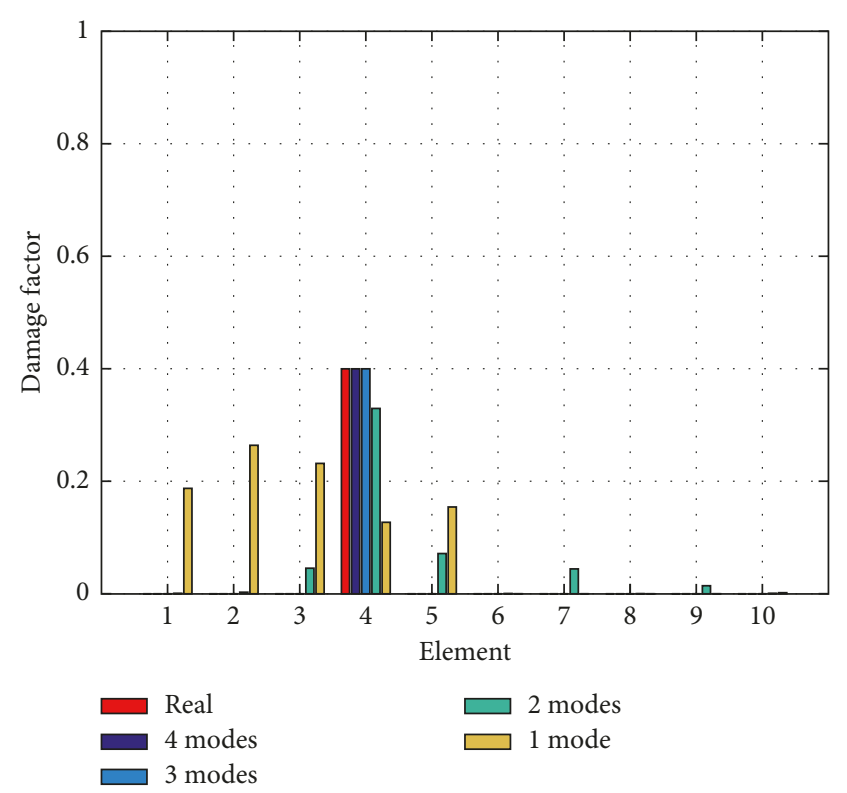

(a)

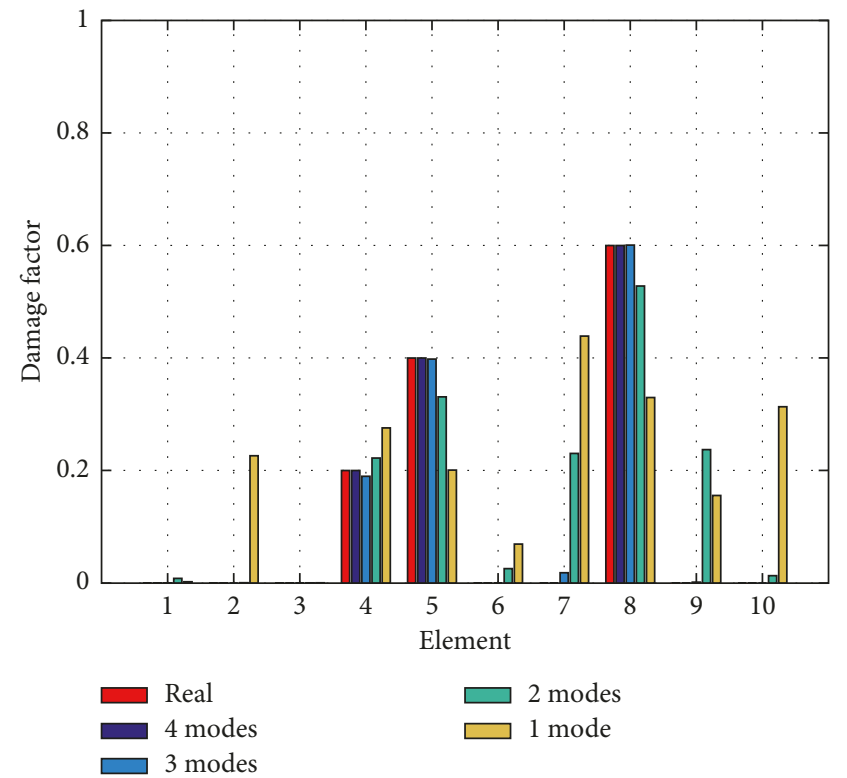

(c)

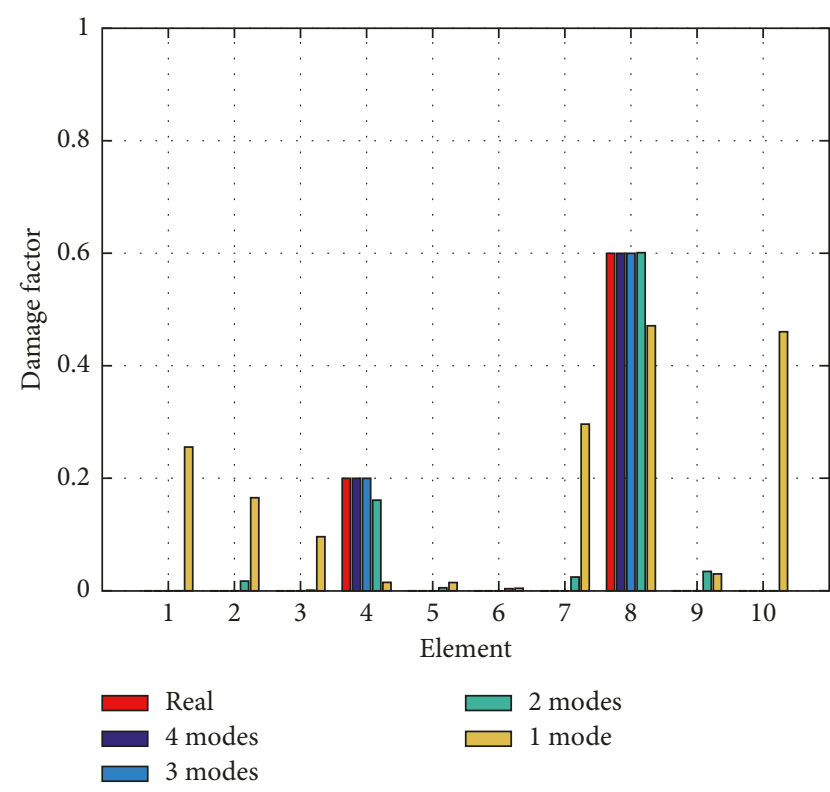

(b)

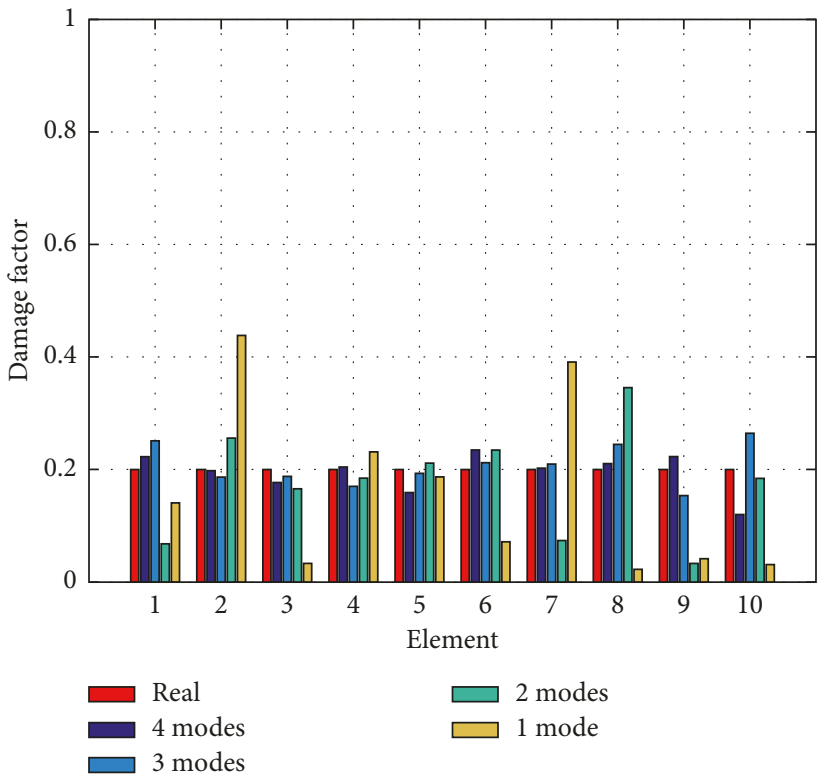

(d)

FIGURE 7: Beam example: performance of the combined criterion for different damage scenarios (noise 5\%). (a) Scenario 1, (b) scenario 2 , (c) scenario 3 , and (d) scenario 4 .

TABLe 2: Beam example: norm values for different damage scenarios and 1 to 4 known modes (noise 5\%).

\begin{tabular}{lcccc}
\hline Known modes & \multicolumn{3}{c}{ Damage scenario } & 3 \\
\hline 1 mode & 1 & 2 & 0.425 & \\
2 modes & 0.137 & 0.370 & 0.093 & 0.307 \\
3 modes & 0.137 & 0.087 & 0.025 & 0.313 \\
4 modes & 0.000 & 0.000 & 0.000 & 0.128 \\
\hline
\end{tabular}

a rectangular cross section of $0.25 \mathrm{~m} \times 0.30 \mathrm{~m}$ with an orientation in favor of inplane bending (the principal moment of inertia $5.625 \cdot 10^{-4} \mathrm{~m}^{4}$ applies to in-plane bending). The material has Young's modulus $E$ equal to $30 \mathrm{GPa}$ and a density $\rho$ equal to $2500 \mathrm{~kg} / \mathrm{m}^{3}$, which applies to the whole structure (beam and columns). 

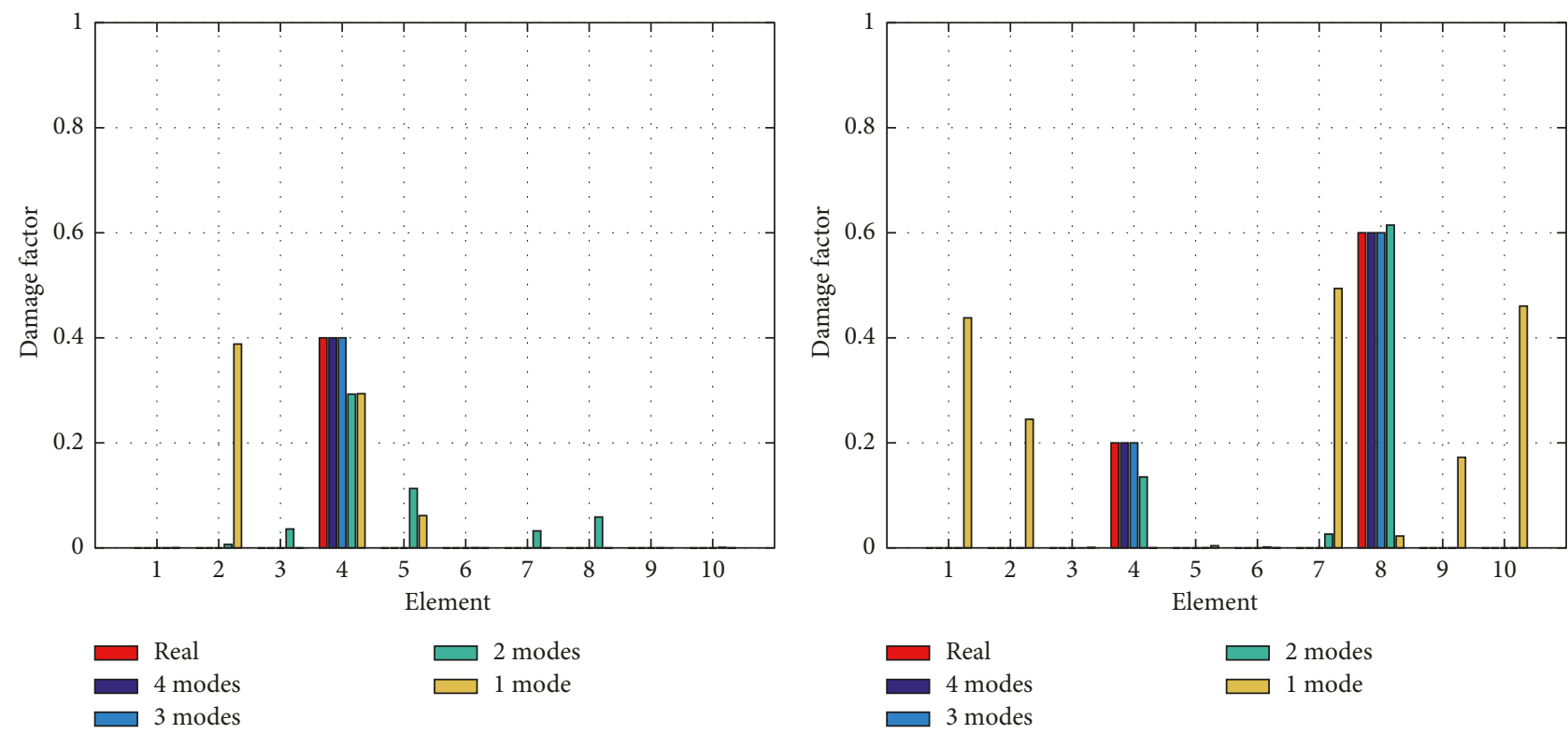

(a)

(b)
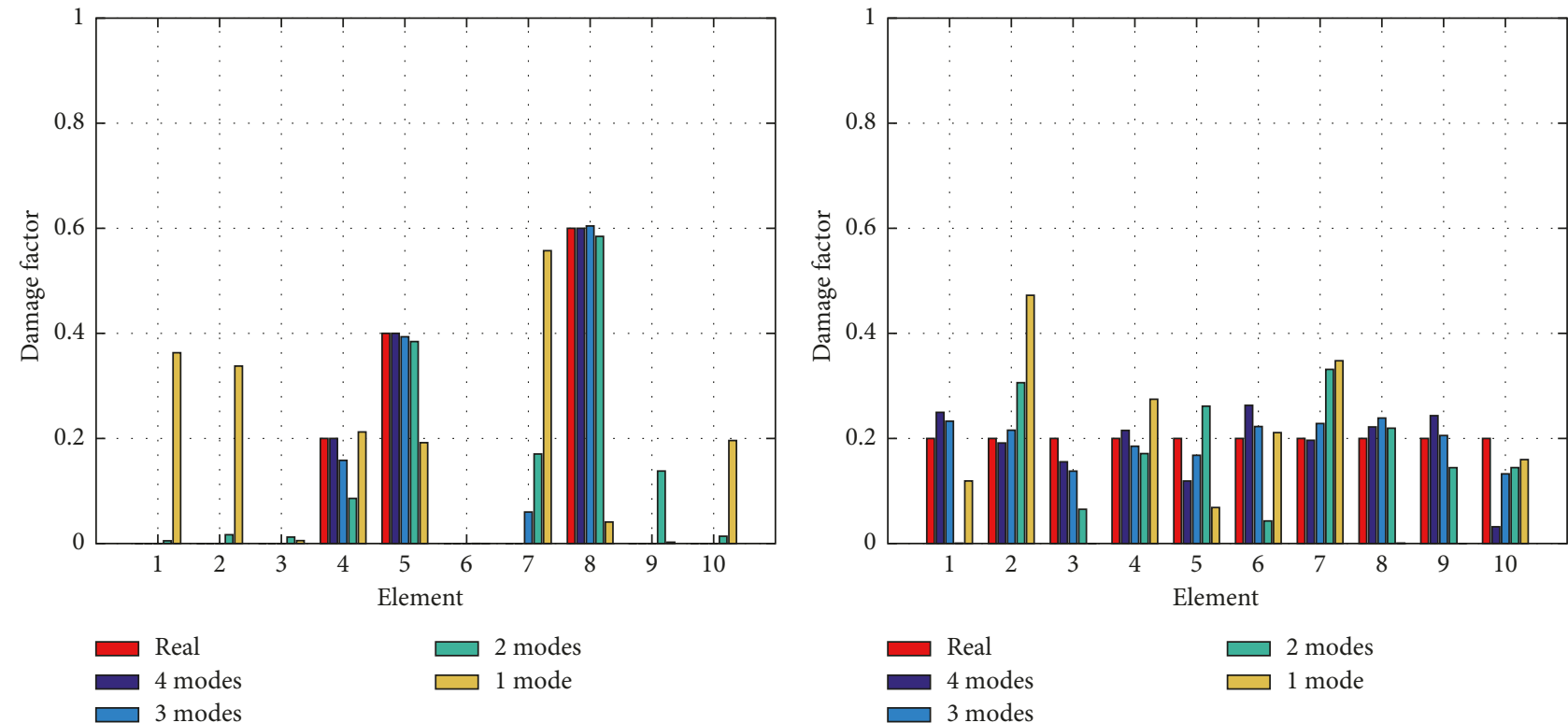

(c)

(d)

FIGURE 8: Beam example: performance of the combined criterion for different damage scenarios (noise 10\%). (a) Scenario 1, (b) scenario 2 , (c) scenario 3 , and (d) scenario 4 .

TABle 3: Beam example: norm values for different damage scenarios and 1 to 4 known modes (noise $10 \%$ ).

\begin{tabular}{|c|c|c|c|c|}
\hline \multirow{2}{*}{ Known modes } & \multicolumn{4}{|c|}{ Damage scenario } \\
\hline & 1 & 2 & 3 & 4 \\
\hline 1 mode & 0.259 & 0.571 & 0.651 & 0.518 \\
\hline 2 modes & 0.119 & 0.059 & 0.110 & 0.353 \\
\hline 3 modes & 0.000 & 0.000 & 0.072 & 0.122 \\
\hline 4 modes & 0.000 & 0.000 & 0.000 & 0.222 \\
\hline
\end{tabular}

The monitored DOFs are all the horizontal DOFs of the two columns, excluding the supports (8 DOFs for each column), and all vertical DOFs of the beam, excluding the corner DOFs (11 DOFs for the beam). The total number of monitored DOFs is thus 27 , and the first four eigenmodes of the undamaged structure are shown in Figure 10. 


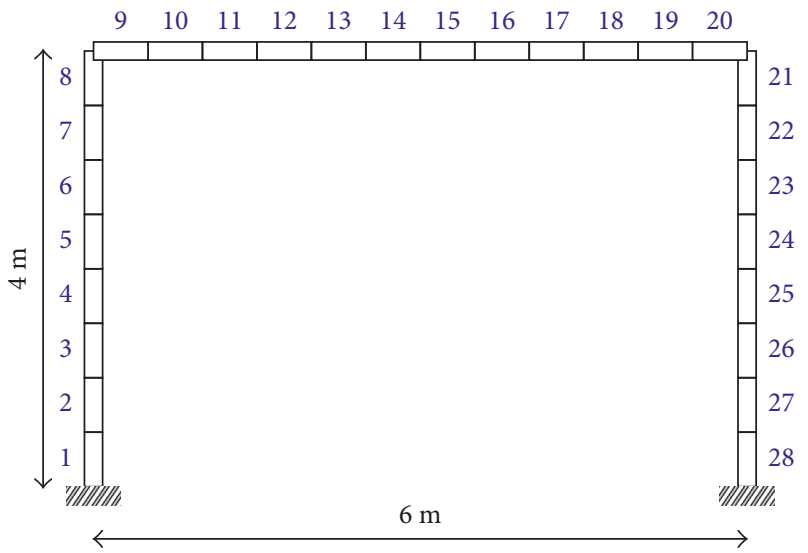

Figure 9: The frame structure under investigation.

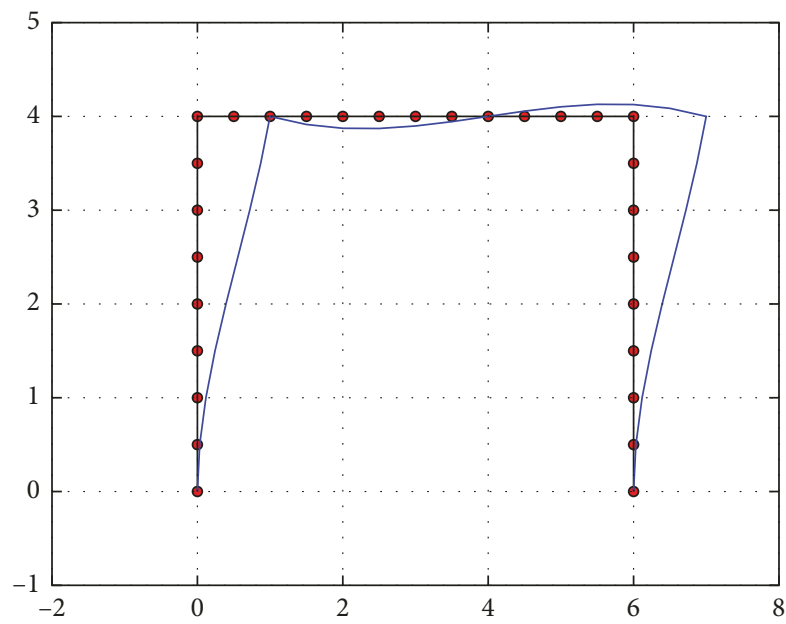

(a)

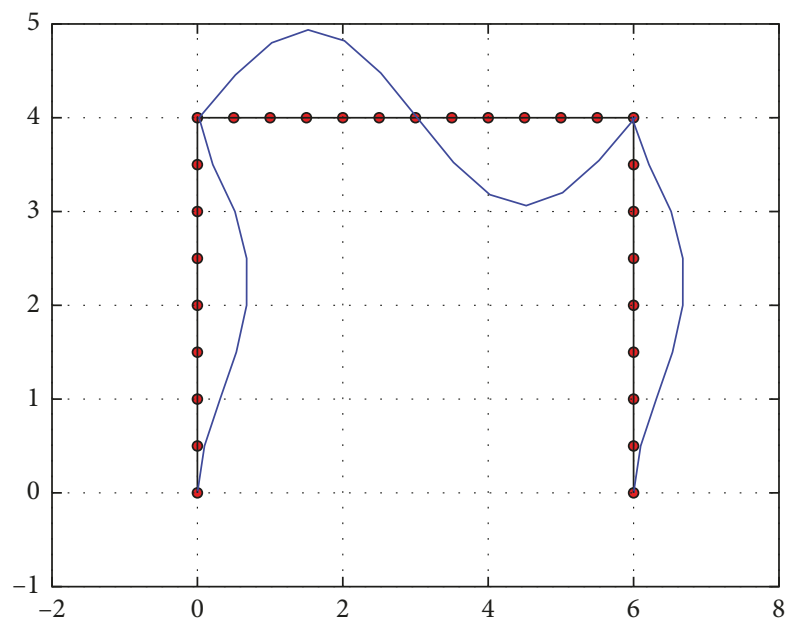

(c)

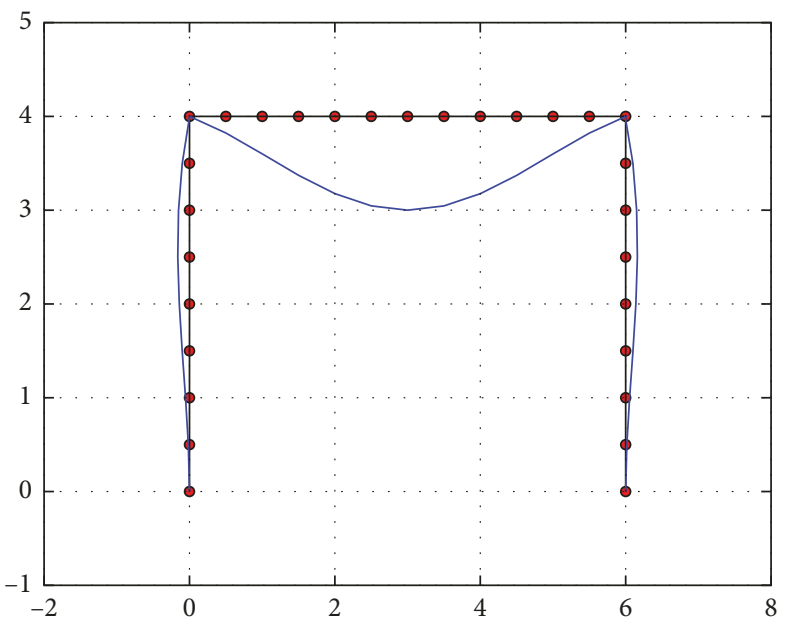

(b)

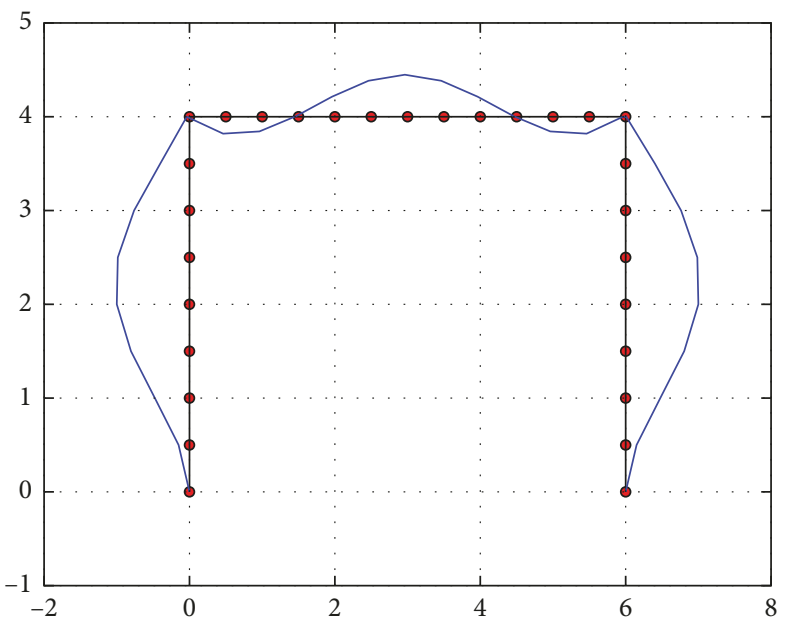

(d)

Figure 10: Frame example: first four eigenmodes of the undamaged structure. (a) Eigenmode $1\left(T_{1}=0.128 \mathrm{~s}\right)$, (b) eigenmode $2\left(T_{2}=0.053 \mathrm{~s}\right)$, (c) eigenmode $3\left(T_{3}=0.021 \mathrm{~s}\right)$, and (d) eigenmode $4\left(T_{4}=0.019 \mathrm{~s}\right)$.

Four different damage scenarios are considered: (a) a single-element damage scenario, (b) a five-element damage scenario along a location on the beam, (c) a five-element damage scenario along a location at the left beam-column connection, and (d) a nine-element damage scenario with unsymmetrical damages at the two corners, as shown 


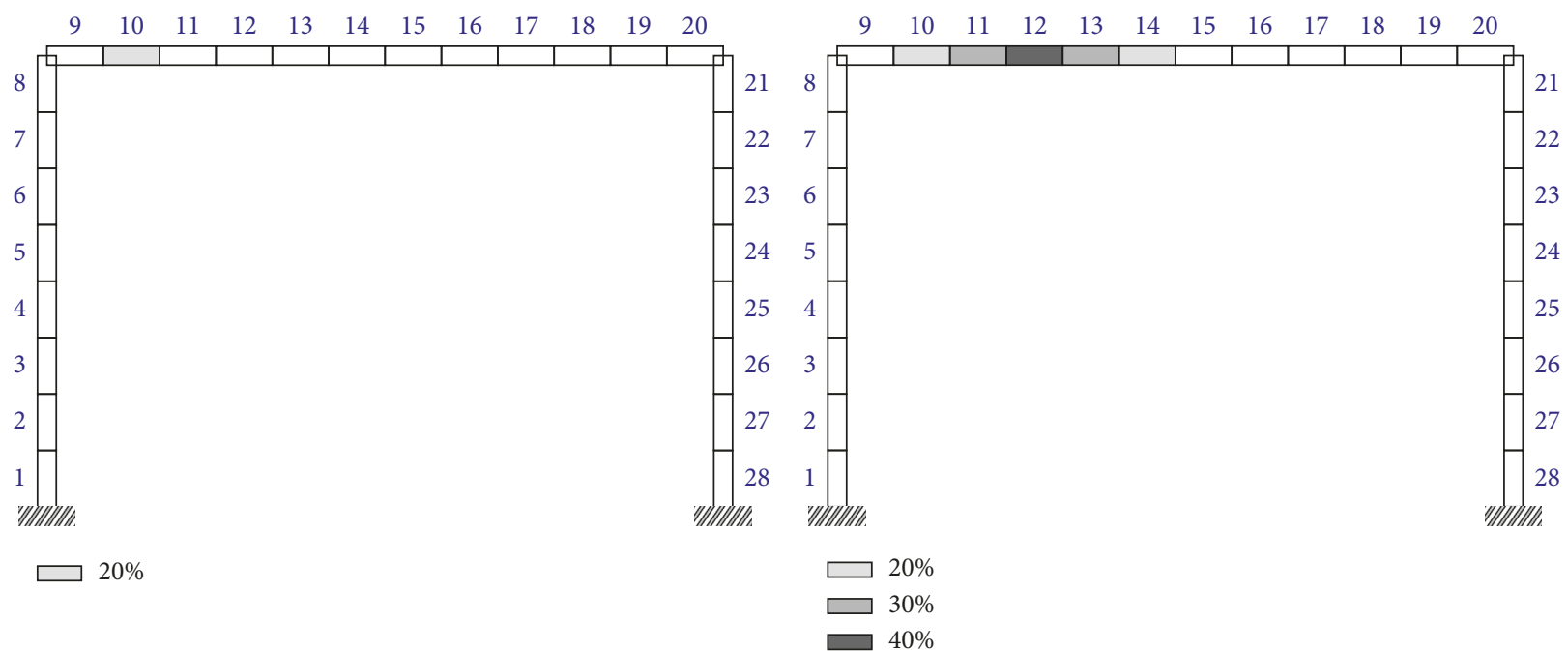

(a)

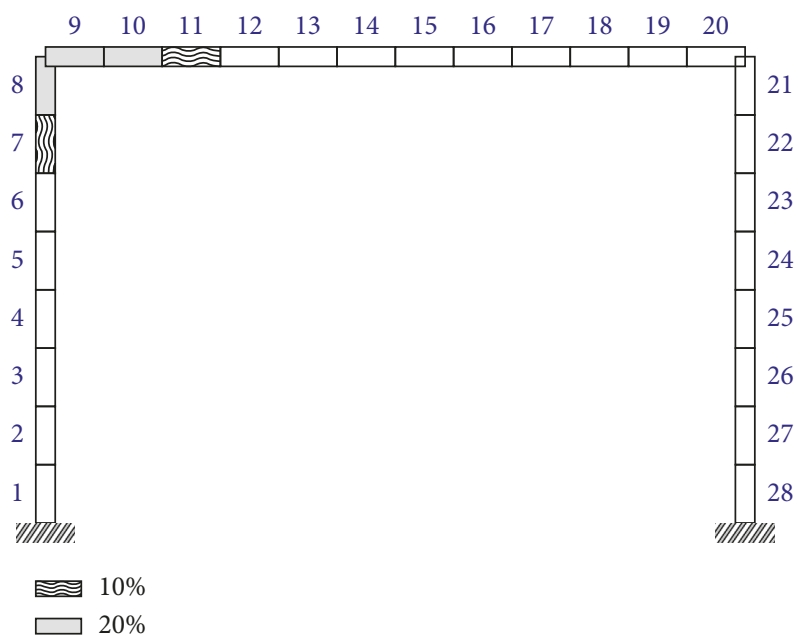

(c) (b)

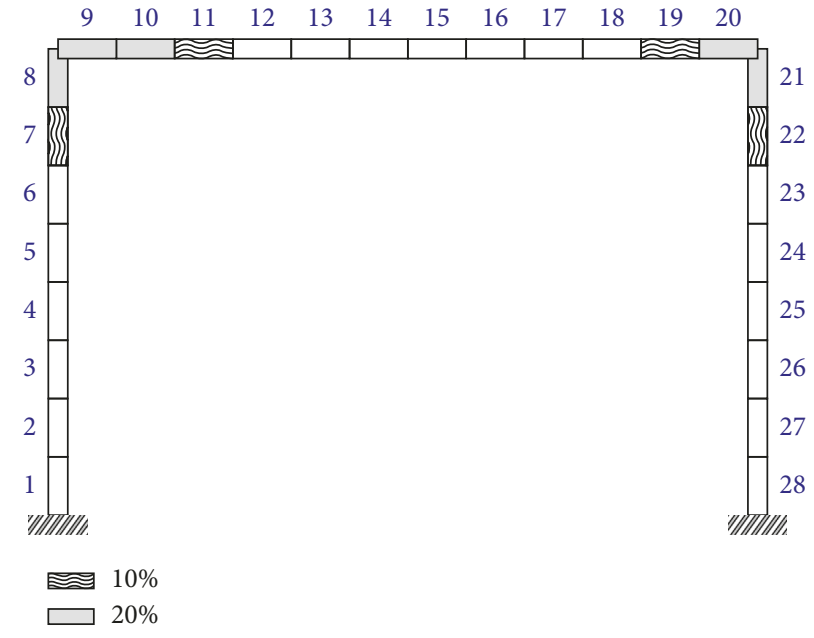

(d)

FIgURE 11: Frame example: the four different damage scenarios. (a) Scenario 1, (b) scenario 2, (c) scenario 3, and (d) scenario 4.

in Figure 11. In this numerical example, all individual criteria, $F_{1}$ (based on MTMAC), $F_{2}$ (based on MACFLEX), and $F$ (combined), are examined and compared to each other.

\subsubsection{No Noise}

(1) MTMAC Criterion Results $\left(F_{1}\right)$. We investigate the performance of the MTMAC criterion for all four damage scenarios. The optimized values of the design variables for the objective function $F_{1}$ are shown in Figure 12, for each damage scenario.

As shown in Figure 12, even in the case of four known eigenmodes, using only the MTMAC criterion does not lead to results of good quality.

(2) MACFLEX Criterion Results $\left(F_{2}\right)$. We investigate the performance of the MACFLEX criterion for all four damage cases. The optimized values of the design variables for the objective function $F_{2}$ are shown in Figure 13, for each damage scenario.

(3) Combined Criterion Results (F). Next, we investigate the performance of the combined criterion for all four damage scenarios. The optimized values of the design variables for the objective function $F$ are shown in Figure 14, for each damage scenario.

Due to the complexity of the specific problem (optimization problem with 28 design variables), it is very difficult to find the exact solution, even in the case of four known eigenmodes. In any case, as shown in Figure 14, using the combined criterion, the optimizer manages to give very good predictions of the location and extent of damage, especially when a larger number of eigenmodes are known.

(4) Combined Criterion versus the Individual Criteria. Figure 15 shows the norm value for each of the three objective functions versus the number of generations, as the optimization problem proceeds, for the second and the third damage 


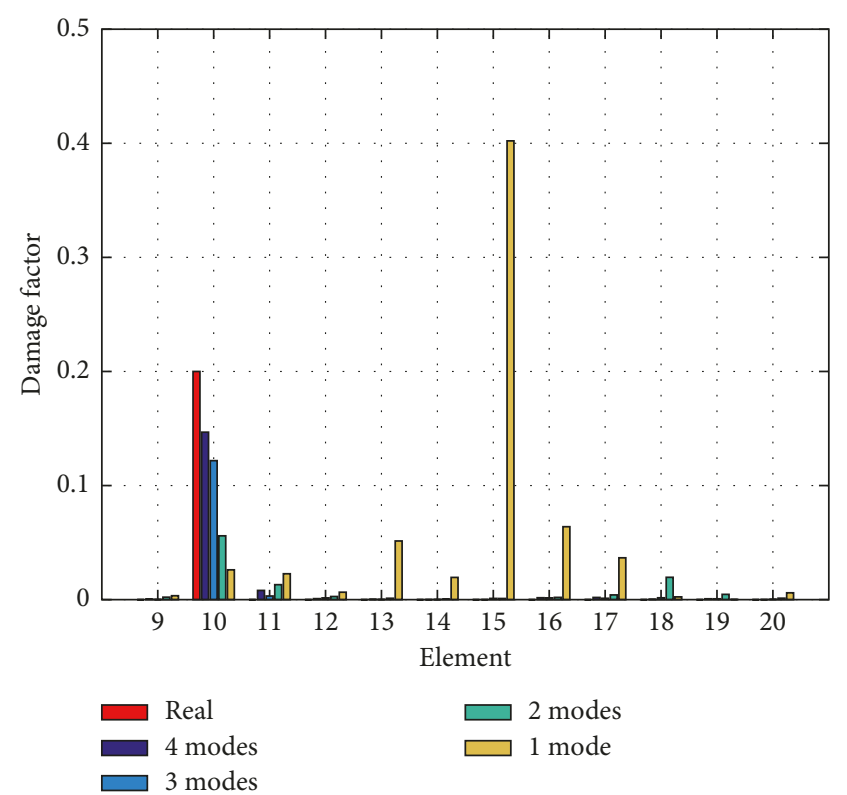

(a)

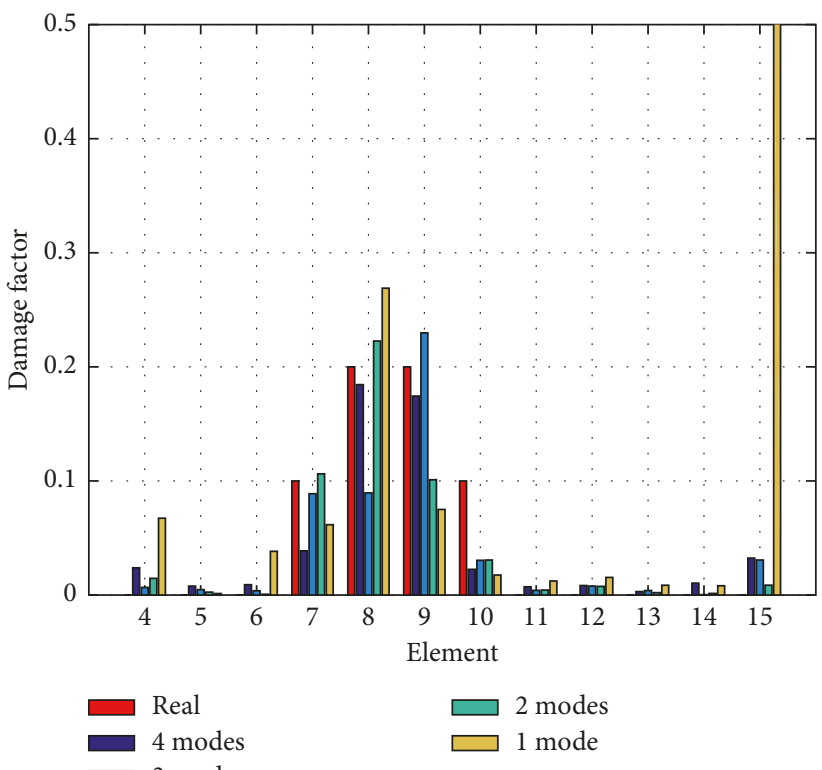

(c)

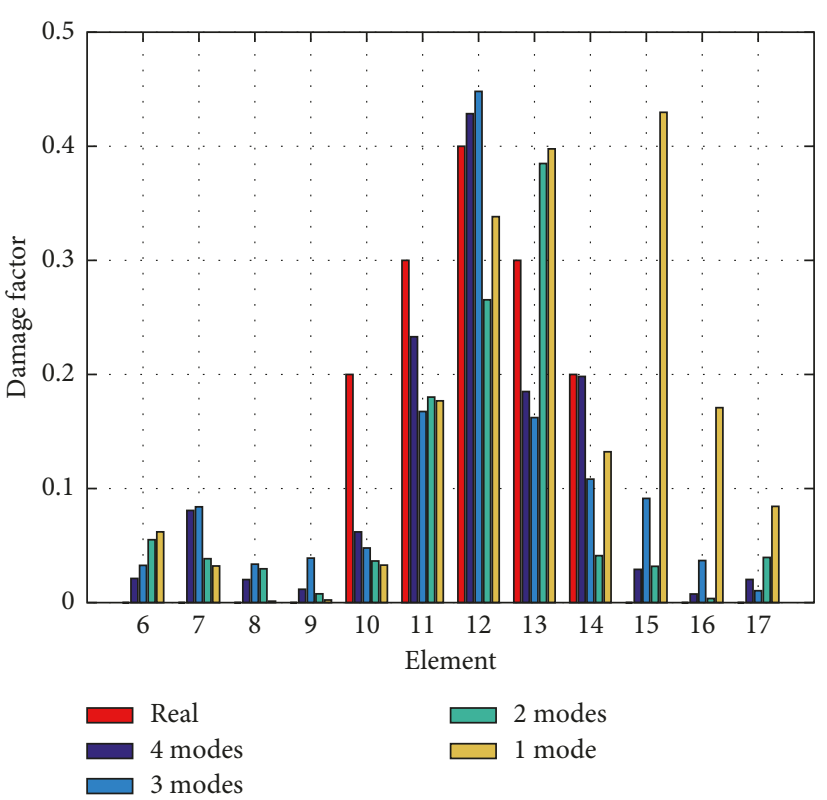

(b)

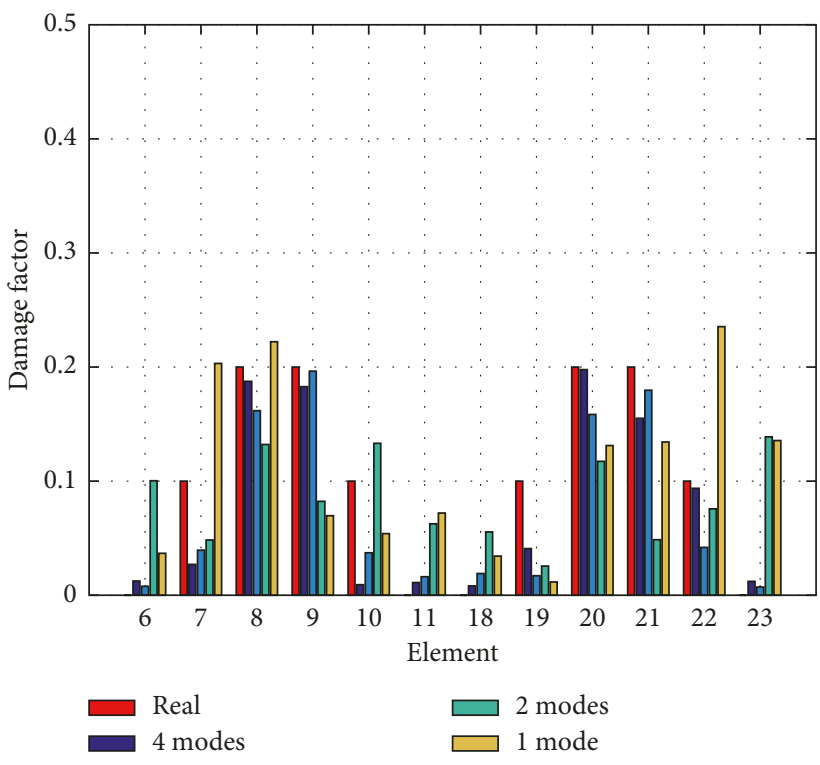

(d)

Figure 12: Frame example: damage prediction of the MTMAC criterion for the four damage scenarios (no noise). (a) Scenario 1, (b) scenario 2, (c) scenario 3, and (d) scenario 4.

scenarios, with three known eigenmodes. The $x$-axis is in logarithmic scale for better clarity of the results.

The MACFLEX criterion $\left(F_{2}\right)$ exhibits the worst performance. Although the performance of the MTMAC criterion $\left(F_{1}\right)$ is good [15], it is shown that the combined criterion $(F)$ exhibits the best performance among the three with faster convergence and more accurate final result for both damage scenarios. Due to space limitations, only the results of scenarios 2 and 3 with three known eigenmodes are presented here, but the trend is the same in all other cases also. This is better highlighted in Table 4, where the norm of the final result is shown for each criterion and each damage scenario (three known eigenmodes). It is clear that the combined criterion shows superior performance managing to converge to the lowest values in each single case.

(5) Influence of the Number of Known Eigenmodes. Figure 16 presents the norm values for the combined objective function $F$ versus the number of generations for damage scenarios 2 and 3, where the different lines represent the different numbers of known eigenmodes (from one to four). Again, the best results are obtained with 4 known eigenmodes, while it is shown that richer modal information leads to better results in general. This was a general observation 


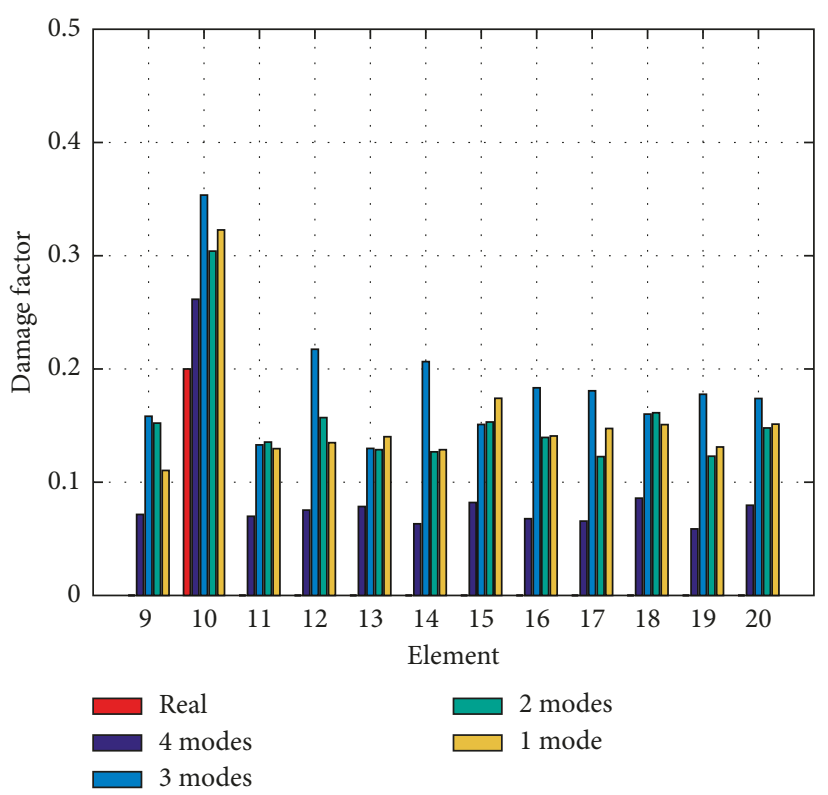

(a)

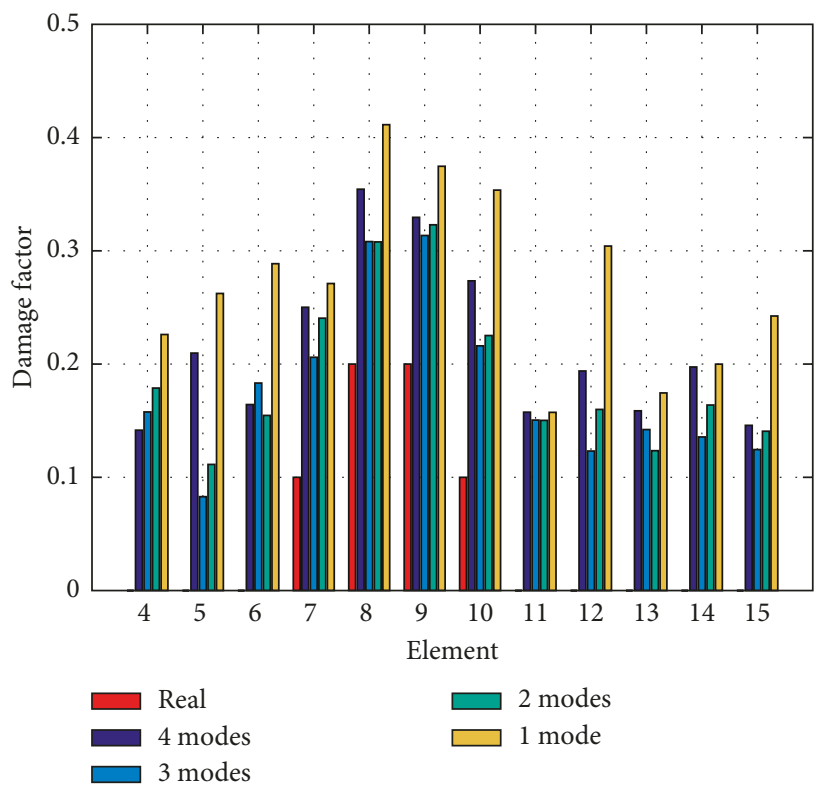

(c)

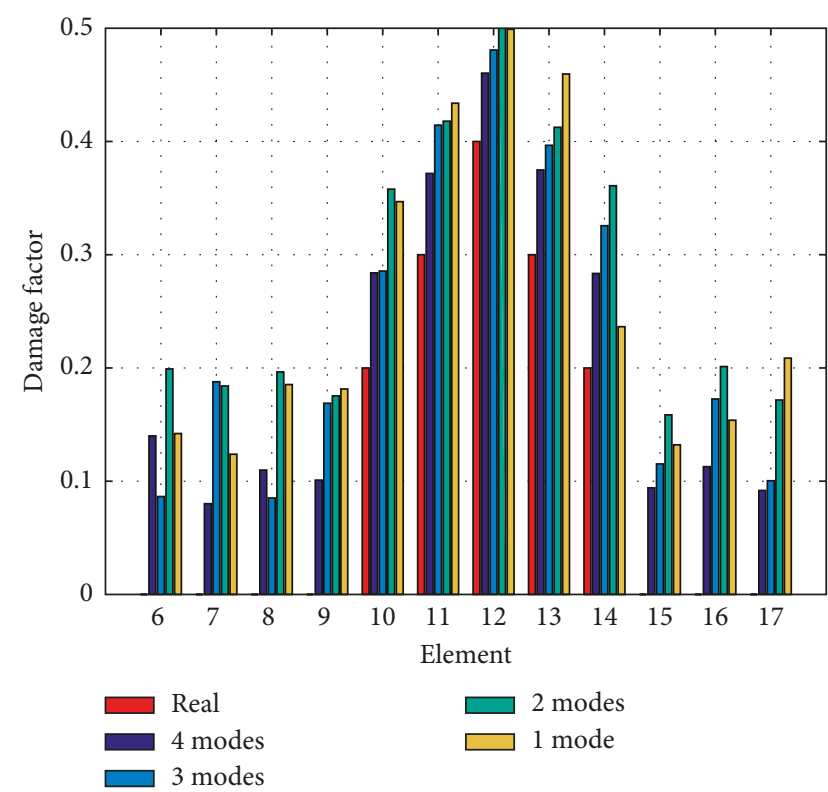

(b)

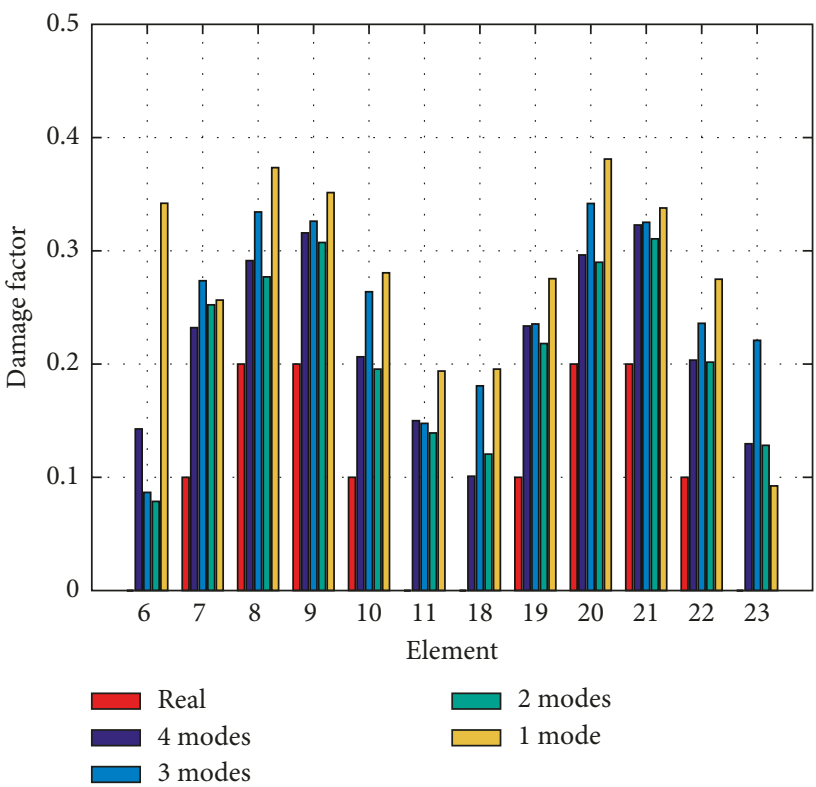

(d)

FIGURE 13: Frame example: damage prediction of the MACFLEX criterion for the four damage scenarios (no noise). Again, the results using the individual MACFLEX criterion are not good, even with four known eigenmodes, let alone cases with less known eigenmodes (1 to 3 ). (a) Scenario 1, (b) scenario 2, (c) scenario 3, and (d) scenario 4.

with other damage scenarios also and for any objective function $\left(F, F_{1}\right.$, and $\left.F_{2}\right)$.

4.2.2. Noise 5\%: Combined Criterion. Noise is added to the experimental data, with noise ratio NR $=5 \%$. In Figure 17, the results of the combined criteria are presented, for all damage scenarios, for different numbers of known eigenmodes (from one to four).

Adding 5\% noise leads to deteriorated results, but again, the optimization procedure manages to give good estimations of the real damage, especially when a higher number of eigenmodes are known.

4.2.3. Noise 10\%: Combined Criterion. At this stage, a different level of noise is added to the experimental data, this time with an increased noise ratio NR $=10 \%$. Figure 18 presents the results of the combined criterion, for all damage scenario cases, for different numbers of known eigenmodes.

Again, noise plays a significant role and deteriorates the quality of the damage prediction. Even with four known 


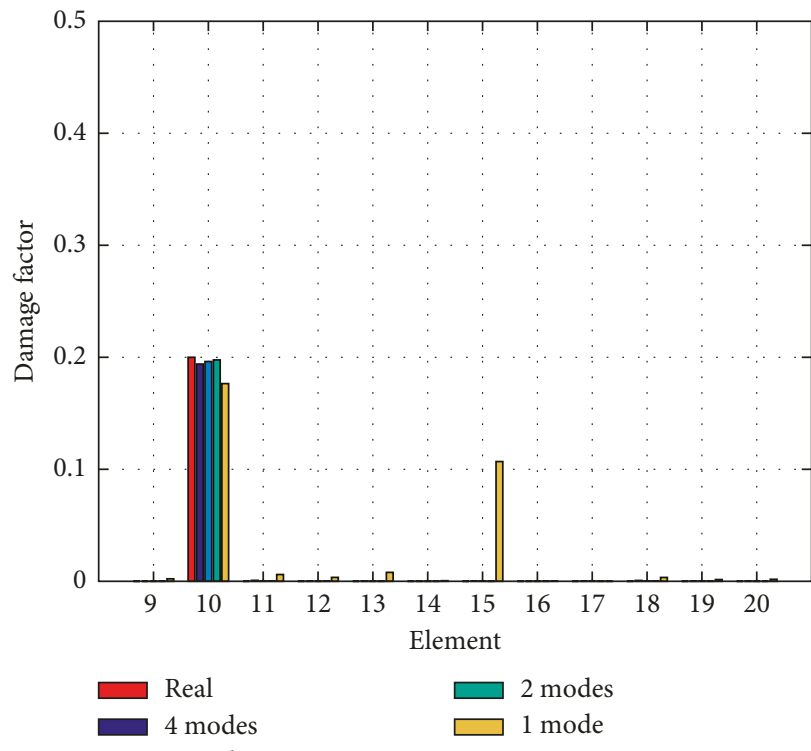

(a)

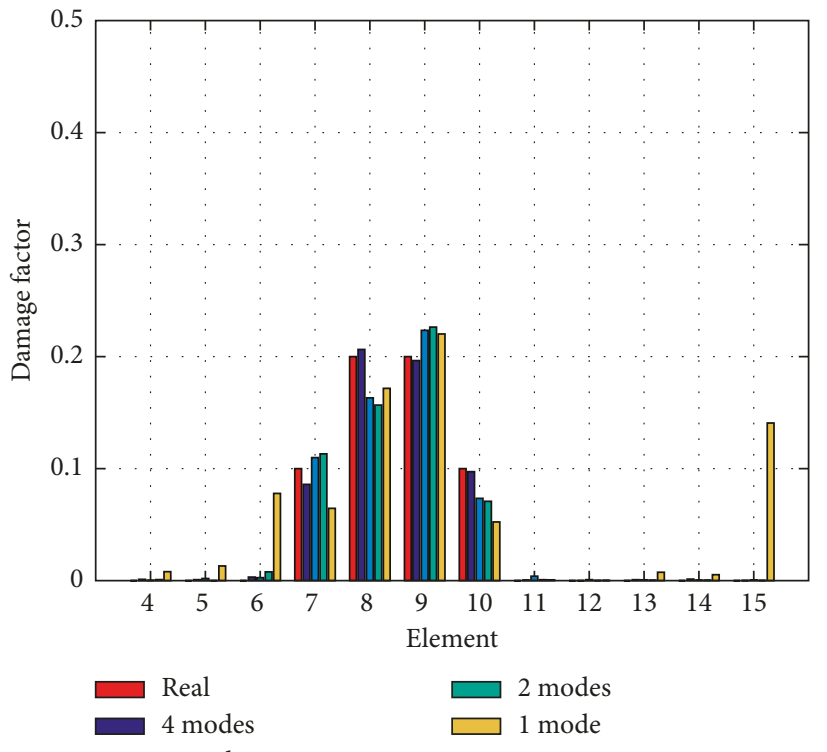

(c)

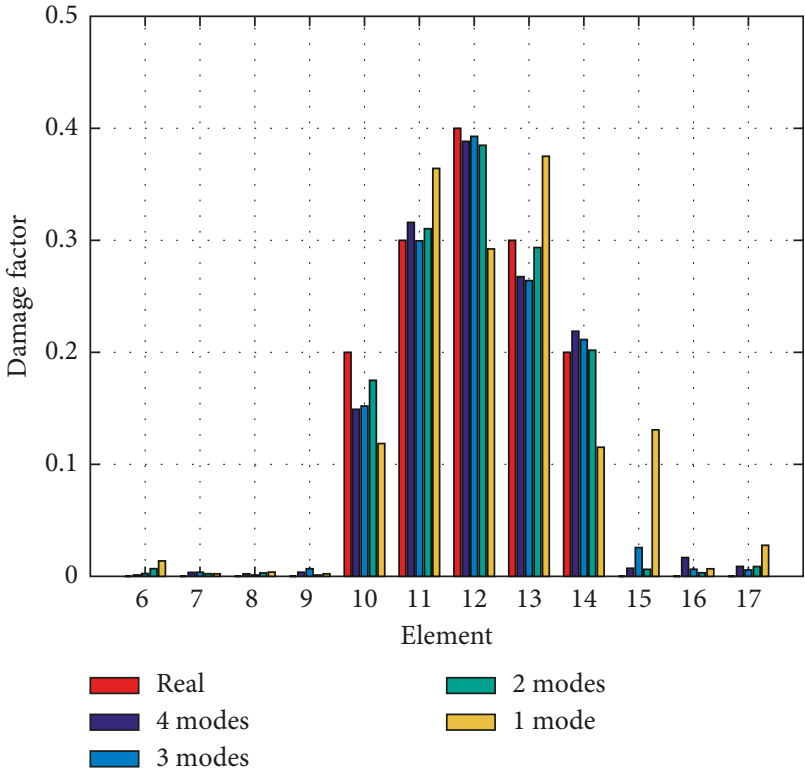

(b)

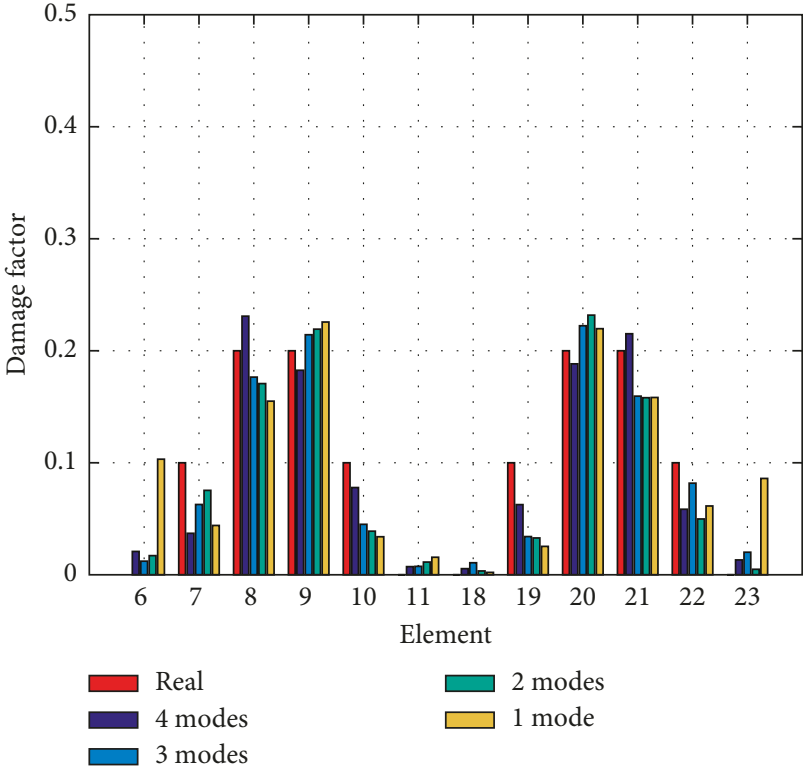

(d)

FIgURE 14: Frame example: damage prediction of the combined criterion for the four damage scenarios (no noise). (a) Scenario 1, (b) scenario 2, (c) scenario 3, and (d) scenario 4.

eigenmodes, the optimizer cannot guarantee great accuracy of the results, except may be for the case of damage scenario 1 , where the performance is still very good. This is normal, taking into account the increased noise ratio in this investigation.

4.2.4. Investigation on the Influence of Noise. Table 5 shows the influence of noise on the final optimization results, where the norm of the final result is shown for each damage scenario without noise and with noise ratios 5\% and $10 \%$ (for three known eigenmodes). It is clear that, for all damage cases, increased noise leads to deteriorated final results.

\section{Conclusions}

In this paper, a new modal correlation criterion is proposed for structural damage identification, which combines two well-known modal correlation criteria, namely, the modal flexibility assurance criterion and the modified total modal assurance criterion. These two criteria take into account vibration characteristics of the structure such as the natural 


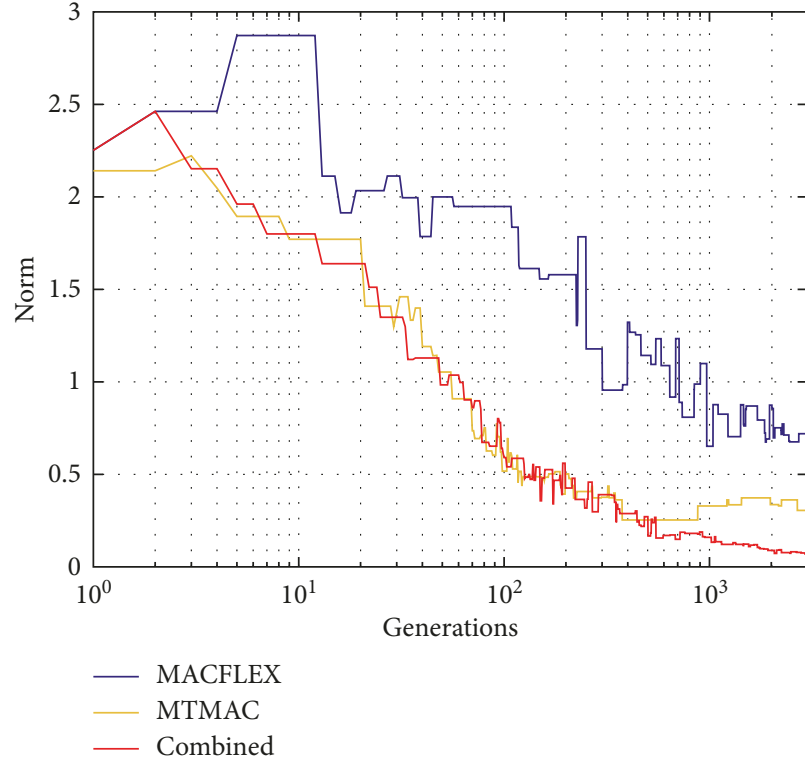

(a)

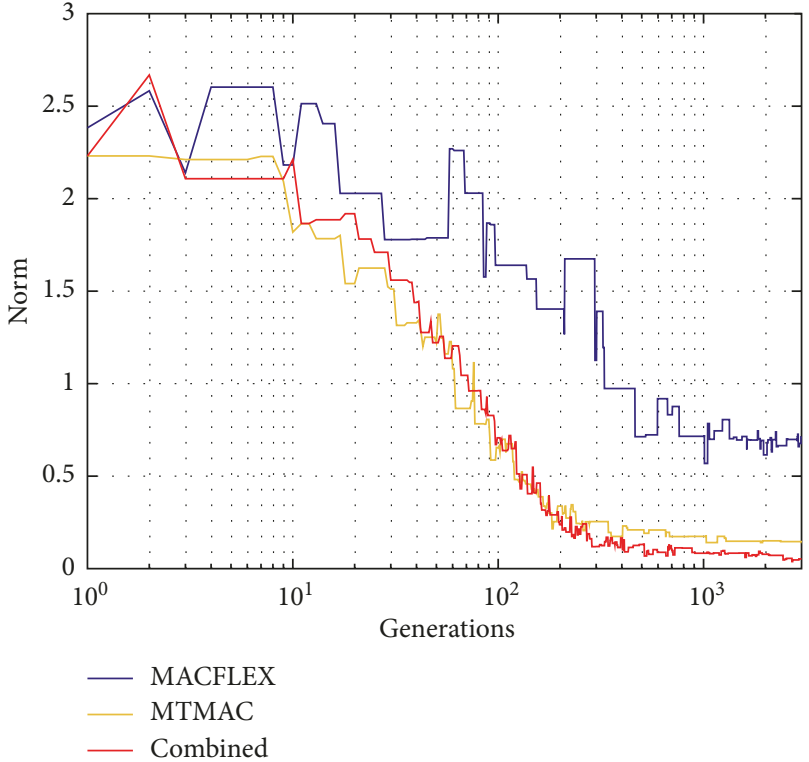

(b)

Figure 15: Frame example: norm values for the three objective functions versus generations, for three known eigenmodes, for damage scenarios 2 and 3 (no noise). (a) Scenario 2 and (b) scenario 3.

TABLE 4: Frame example: norm values for different criteria and damage scenarios (no noise, 3 known eigenmodes).

\begin{tabular}{lcccc}
\hline Criterion & \multicolumn{3}{c}{ Damage scenario } & 3 \\
\hline MTMAC $\left(F_{1}\right)$ & 1 & 2 & 0.142 & 0.183 \\
MACFLEX $\left(F_{2}\right)$ & 0.090 & 0.305 & 0.712 & 0.843 \\
Combined $(F)$ & 0.918 & 0.720 & 0.052 & 0.123 \\
\hline
\end{tabular}

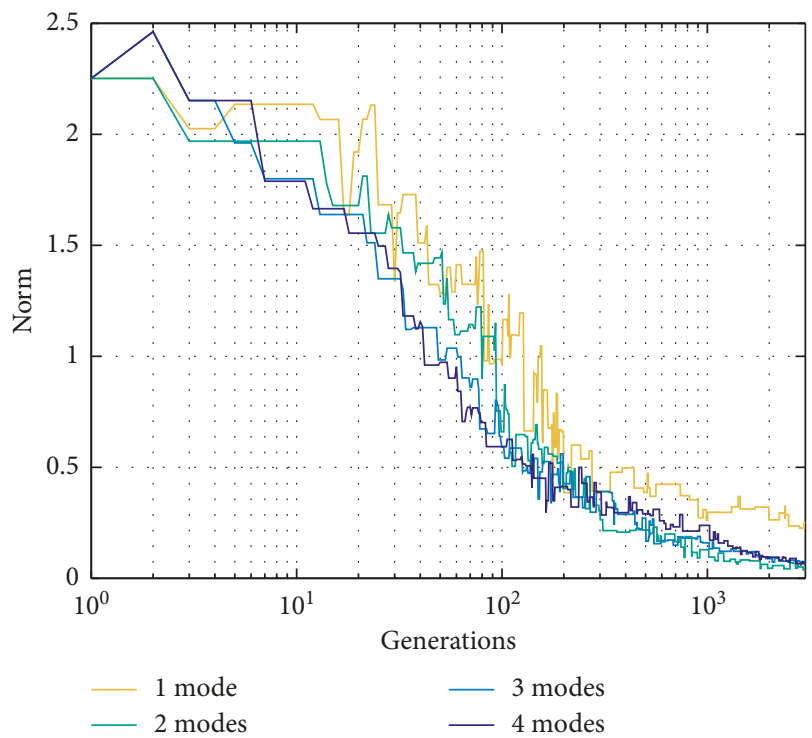

(a)

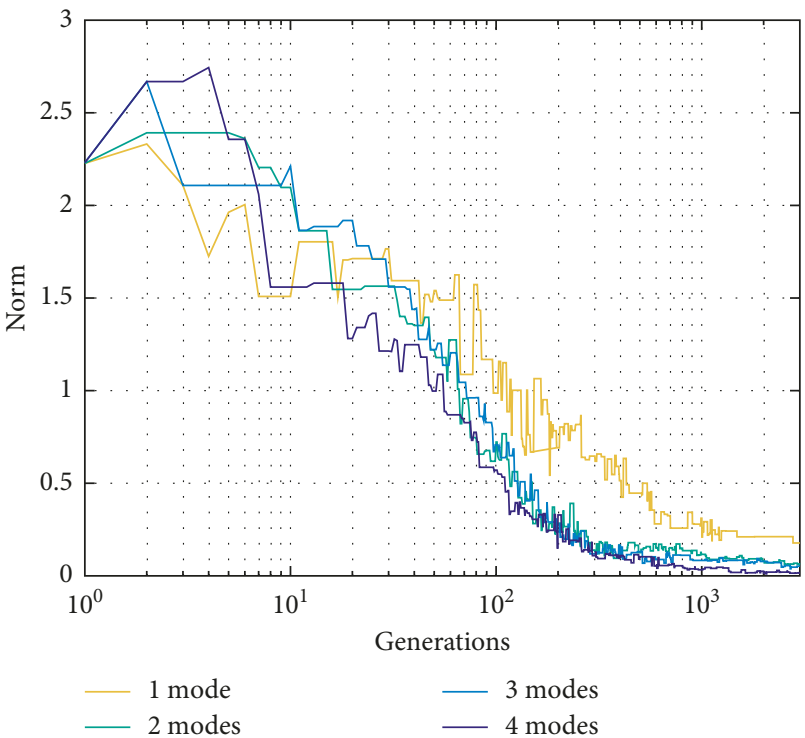

(b)

FIGURE 16: Frame example: norm values for the combined criterion, for different numbers of known eigenmodes, for damage scenarios 2 and 3 (no noise). (a) Scenario 2 and (b) scenario 3. 


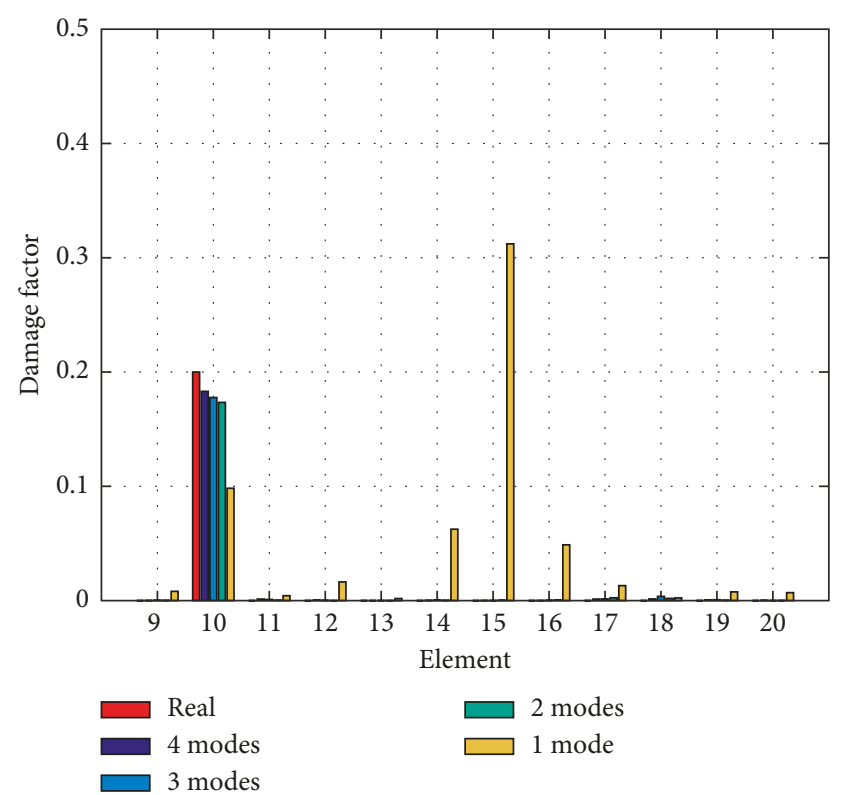

(a)

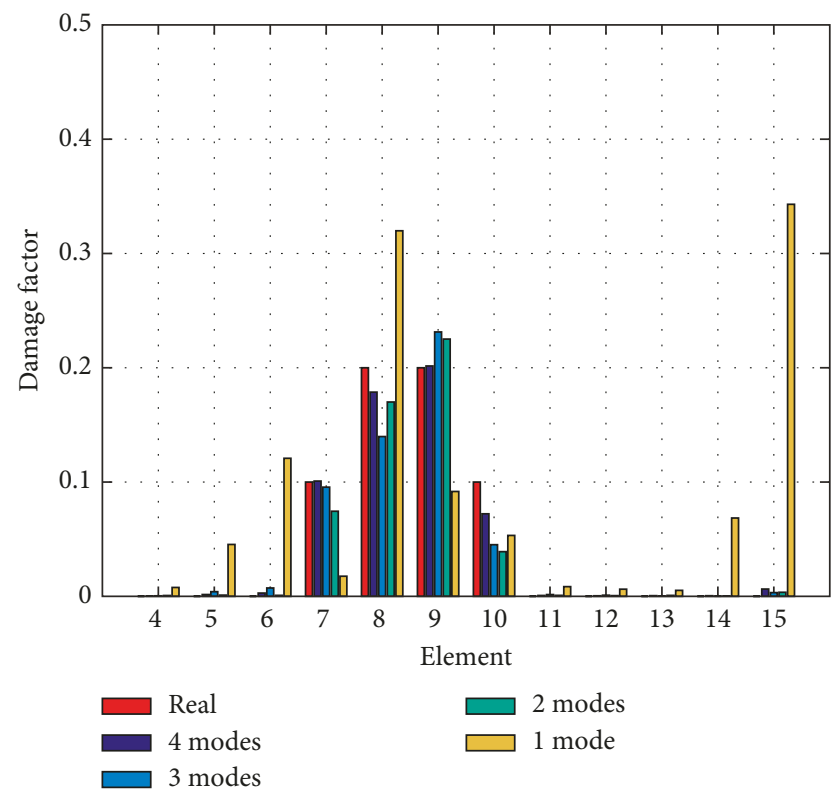

(c)

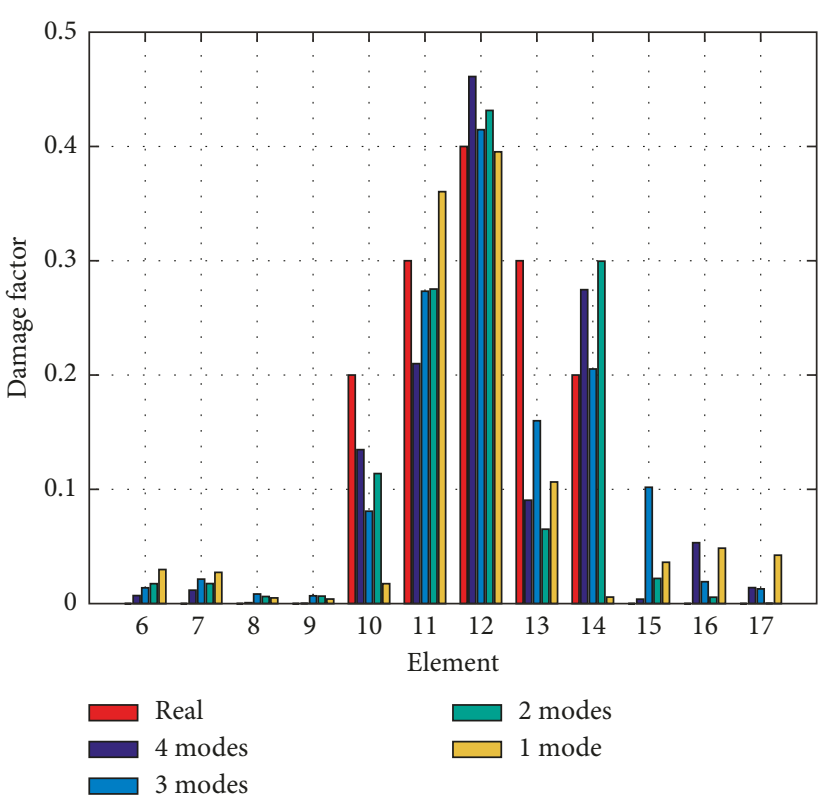

(b)

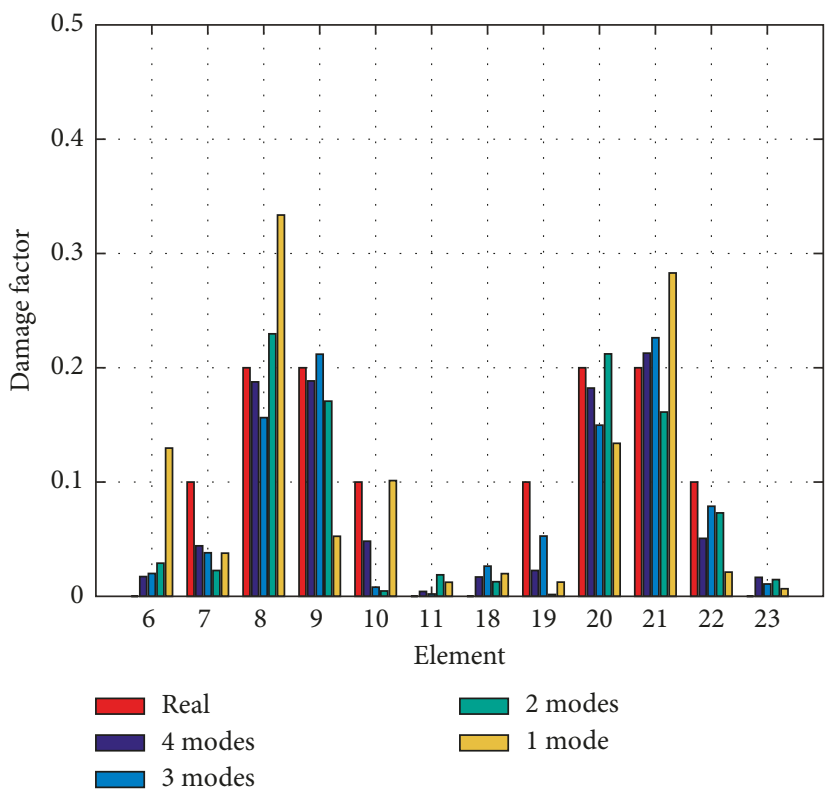

(d)

FIGURE 17: Frame example: performance of the combined criterion for different damage scenarios (noise 5\%). (a) Scenario 1, (b) scenario 2 , (c) scenario 3 , and (d) scenario 4 .

frequencies, the mode shapes, and the modal flexibilities. It was shown that the new combined criterion exhibits optimal performance when compared to the performance of any of the two individual criteria, managing to identify damage in structural elements both in location and extent with very good accuracy.

Due to lack of experimental data, various damage scenarios were assumed in order to simulate possible damage states of the structures considered. Furthermore, to simulate real measured experimental values of modal parameters, artificial noise has been added to the experimental data. In the cases where noisy modal data were considered, the combined criterion also showed an acceptable performance, especially in cases where a higher number of eigenmodes (three or four) were taken into account.

The structural damage identification problem was treated as an optimization problem which was solved using the differential evolution algorithm. The optimization algorithm proved to be very efficient and robust, managing to give results of good quality in the numerical examples considered, especially in cases without the presence of noise.

Some damage scenarios proved to be more difficult than others. Even for the most difficult damage scenarios (i.e., the fourth scenario in both numerical examples), the combined 


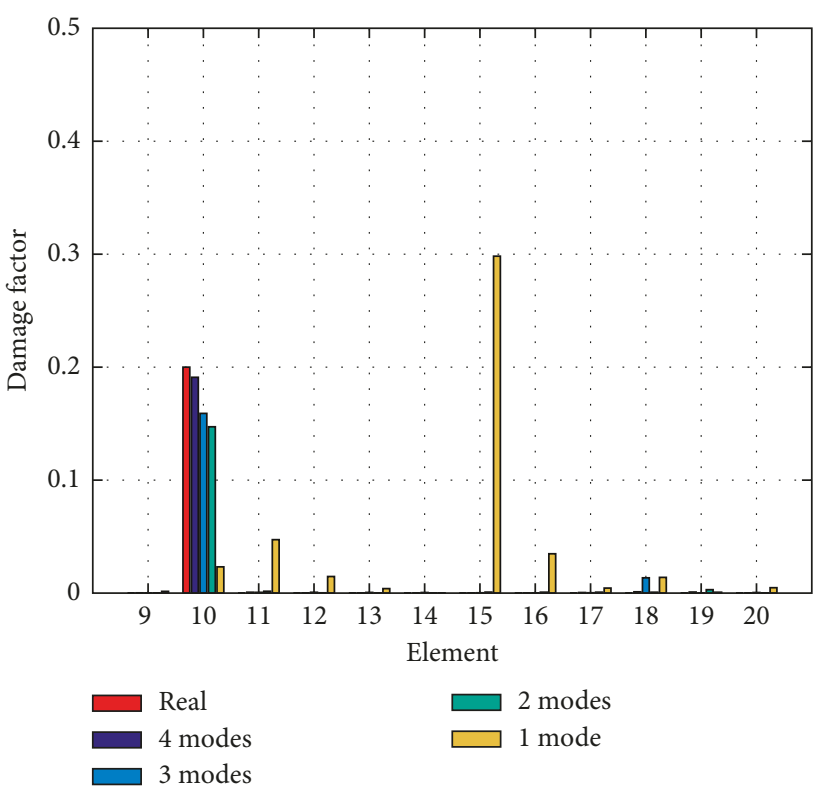

(a)

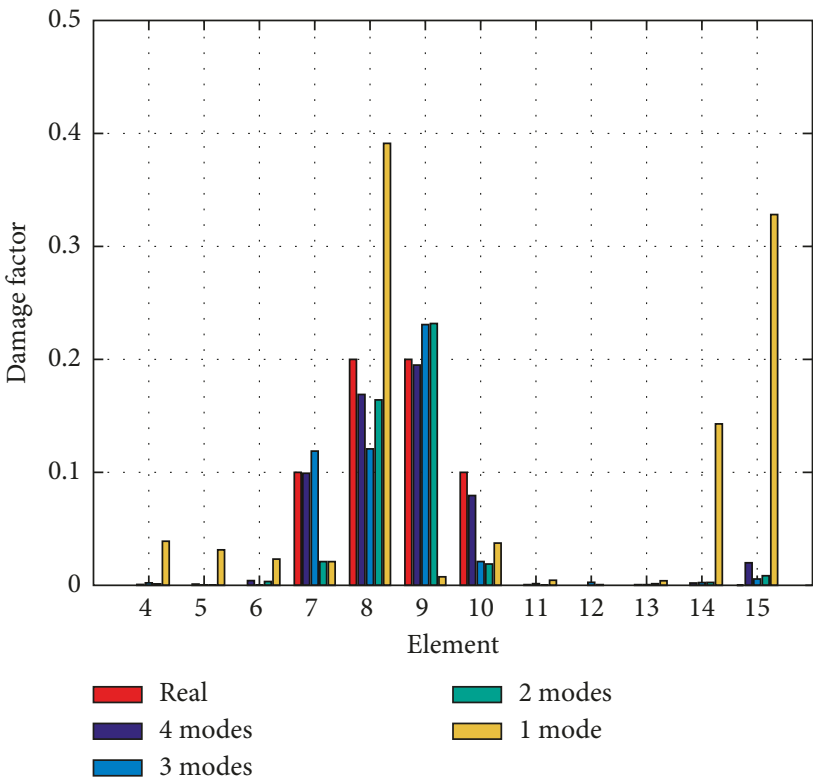

(c)

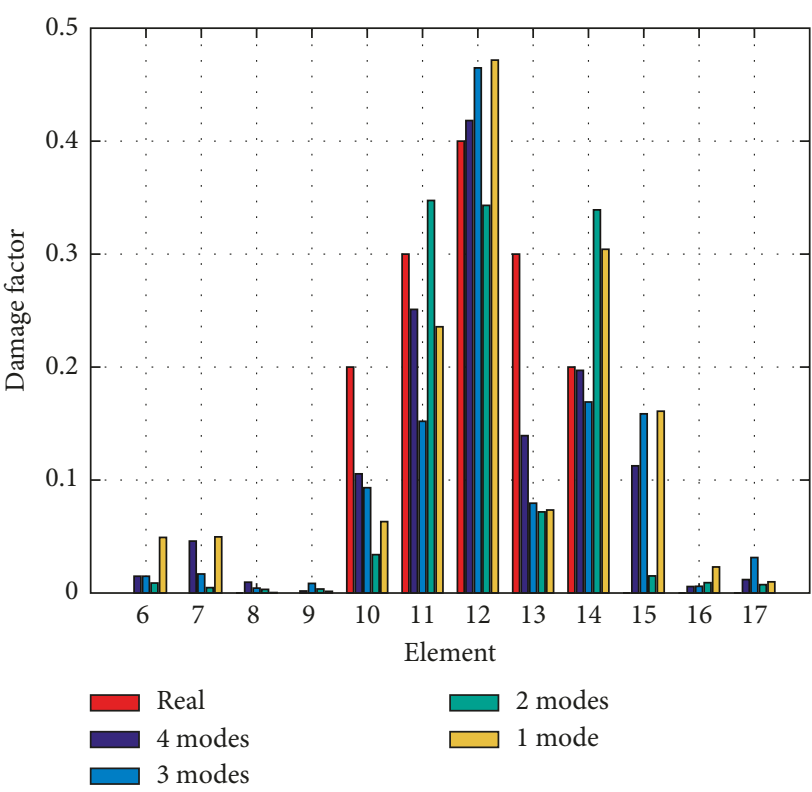

(b)

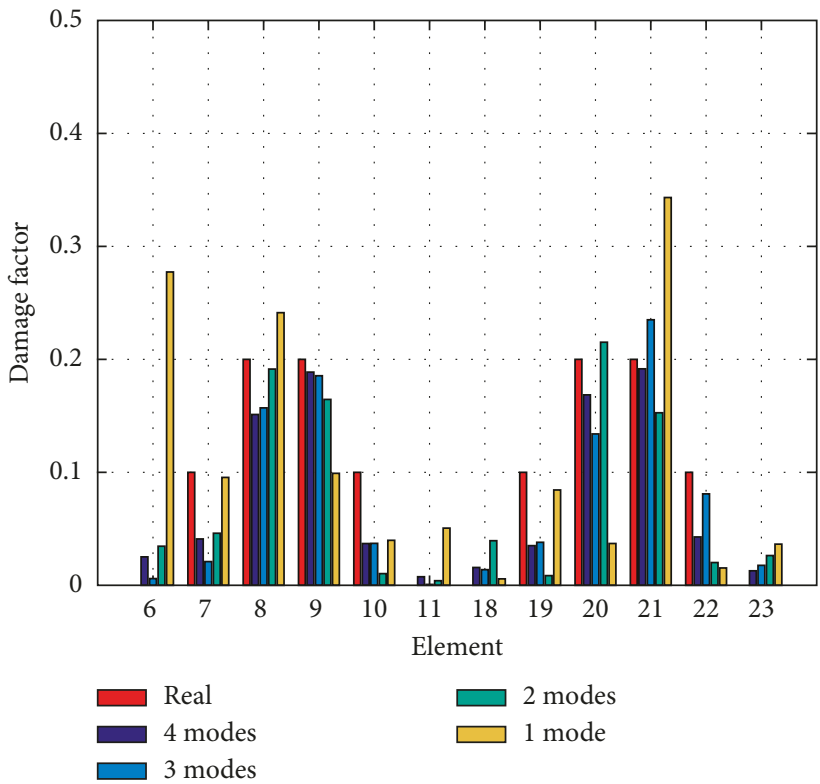

(d)

FiguRE 18: Frame example: performance of the combined criterion for different damage scenarios (noise 10\%). (a) Scenario 1, (b) scenario 2, (c) scenario 3 , and (d) scenario 4 .

TABle 5: Frame example: norm values for the combined criterion and different damage scenarios (three known eigenmodes).

\begin{tabular}{|c|c|c|c|c|}
\hline \multirow{2}{*}{ Criterion } & \multicolumn{4}{|c|}{ Damage scenario } \\
\hline & 1 & 2 & 3 & 4 \\
\hline No noise & 0.004 & 0.073 & 0.052 & 0.123 \\
\hline Noise $5 \%$ & 0.024 & 0.220 & 0.091 & 0.165 \\
\hline Noise $10 \%$ & 0.047 & 0.344 & 0.121 & 0.199 \\
\hline
\end{tabular}

criterion managed to give a very good estimation of the extent and location of damage. However, in these cases, a higher number of eigenmodes were needed in order to achieve a solution of acceptable quality, especially when noise was present.
The presence of noise can affect the performance of the damage identification procedure, especially with increasing noise intensities and, therefore, it is a way of evaluating the robustness of the proposed methodology. It is impressive 
that the combined criterion exhibits better performance than any of the two individual criteria, even when there is no noise for them, in comparison to the combined criterion with noisy data.

\section{Conflicts of Interest}

The authors declare that there are no conflicts of interest regarding the publication of this article.

\section{Acknowledgments}

This work has been supported by the European Research Council Advanced Grant MASTER-Mastering the Computational Challenges in Numerical Modeling and Optimum Design of CNT Reinforced Composites (ERC-2011-ADG20110209).

\section{References}

[1] S. Laflamme, L. Cao, E. Chatzi, and F. Ubertini, "Damage detection and localization from dense network of strain sensors," Shock and Vibration, vol. 2016, Article ID 2562949, 13 pages, 2016.

[2] K. K. Walsh, B. T. Kelly, and E. P. Steinberg, "Damage identification for prestressed adjacent box-beam bridges," Advances in Civil Engineering, vol. 2014, Article ID 540363, 16 pages, 2014.

[3] H. Liu, G. Song, Y. Jiao, P. Zhang, and X. Wang, "Damage identification of bridge based on modal flexibility and neural network improved by particle swarm optimization," Mathematical Problems in Engineering, vol. 2014, Article ID 640925, 8 pages, 2014.

[4] A. Majumdar, B. Nanda, D. K. Maiti, and D. Maity, "Structural damage detection based on modal parameters using continuous ant colony optimization," Advances in Civil Engineering, vol. 2014, Article ID 174185, 14 pages, 2014.

[5] W. Zheng and Y. Yu, "Bayesian probabilistic framework for damage identification of steel truss bridges under joint uncertainties," Advances in Civil Engineering, vol. 2013, Article ID 307171, 13 pages, 2013.

[6] X. Wang, C.-C. Chang, and L. Fan, "Nondestructive damage detection of bridges: a status review," Advances in Structural Engineering, vol. 4, no. 2, pp. 75-91, 2001.

[7] M. I. Friswell and J. Mottershead, Finite Element Model Updating in Structural Dynamics, Springer, Netherlands, 1995.

[8] J. E. Mottershead and M. I. Friswell, "Model updating in structural dynamics: a survey," Journal of Sound and Vibration, vol. 167, no. 2, pp. 347-375, 1993.

[9] J.-H. Chou and J. Ghaboussi, "Genetic algorithm in structural damage detection," Computers \& Structures, vol. 79, no. 14, pp. 1335-1353, 2001.

[10] C. G. Koh and M. J. Perry, "Structural identification and damage detection using genetic algorithms," Structures and Infrastructures, vol. 6, CRC Press, Boca Raton, FL, USA, 2009.

[11] R. I. Levin and N. A. J. Lieven, "Dynamic finite element model updating using simulated annealing and genetic algorithms," Mechanical Systems and Signal Processing, vol. 12, no. 1, pp. 91-120, 1998.

[12] T. Marwala, Finite Element Model Updating Using Computational Intelligence Techniques: Applications to Structural
Dynamics, Springer Science \& Business Media, Berlin, Germany, 2010.

[13] M. Nobahari and S. M. Seyedpoor, "Structural damage detection using an efficient correlation-based index and a modified genetic algorithm," Mathematical and Computer Modelling, vol. 53, no. 9-10, pp. 1798-1809, 2011.

[14] R. Perera, A. Ruiz, and C. Manzano, "An evolutionary multiobjective framework for structural damage localization and quantification," Engineering Structures, vol. 29, no. 10, pp. 2540-2550, 2007.

[15] M. Georgioudakis and V. Plevris, "Investigation of the performance of various modal correlation criteria in structural damage identification," in Proceedings of the VII European Congress on Computational Methods in Applied Sciences and Engineering, pp. 5626-5645, Crete Island, Greece, June 2016.

[16] A. Rytter, Vibrational Based Inspection of Civil Engineering Structures, Aalborg University, Denmark, 1993.

[17] L. Yu and X. Chen, "Bridge damage identification by combining modal flexibility and PSO methods," in Proceedings of the 2010 Prognostics and System Health Management Conference, Macau, China, January 2010.

[18] R. J. Allemang, "The modal assurance criterion-twenty years of use and abuse," Sound and Vibration, vol. 37, no. 8, pp. 14-23, 2003.

[19] M. Pastor, M. Binda, and T. Harčarik, "Modal assurance criterion,” Procedia Engineering, vol. 48, pp. 543-548, 2012.

[20] R. Perera and R. Torres, "Structural damage detection via modal data with genetic algorithms," Journal of Structural Engineering (ASCE), vol. 132, no. 9, pp. 1491-1501, 2006.

[21] G.-D. Zhou, T.-H. Yi, H. Zhang, and H.-N. Li, "A comparative study of genetic and firefly algorithms for sensor placement in structural health monitoring," Shock and Vibration, vol. 2015, Article ID 518692, 10 pages, 2015.

[22] D. Bernal, "Extracting flexibility matrices from state-space realizations," in Proceedings of the European COST F3 Conference on System Identification and Structural Health Monitoring, pp. 127-135, Madrid, Spain, 2000.

[23] F. E. Udwadia, "Structural identification and damage detection from noisy modal data," Journal of Aerospace Engineering, vol. 18, no. 3, pp. 179-187, 2005.

[24] N.-D. Hoang, Q.-L. Nguyen, and Q.-N. Pham, "Optimizing construction project labor utilization using differential evolution: a comparative study of mutation strategies," Advances in Civil Engineering, vol. 2015, Article ID 108780, 8 pages, 2015.

[25] R. Storn and K. Price, "Differential evolution-a simple and efficient heuristic for global optimization over continuous spaces," Journal of Global Optimization, vol. 11, no. 4, pp. 341-359, 1997.

[26] S. Das and P. Suganthan, "Differential evolution: a survey of the state-of-the-art," IEEE Transactions on Evolutionary Computation, vol. 15, no. 1, pp. 4-31, 2011.

[27] R. Perera, R. Marin, and A. Ruiz, "Static-dynamic multi-scale structural damage identification in a multi-objective framework," Journal of Sound and Vibration, vol. 332, no. 6, pp. 1484-1500, 2013. 


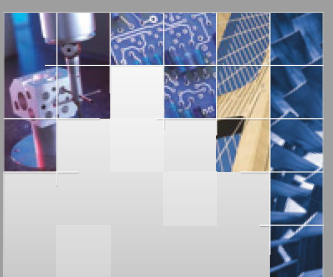

\section{Enfincering}
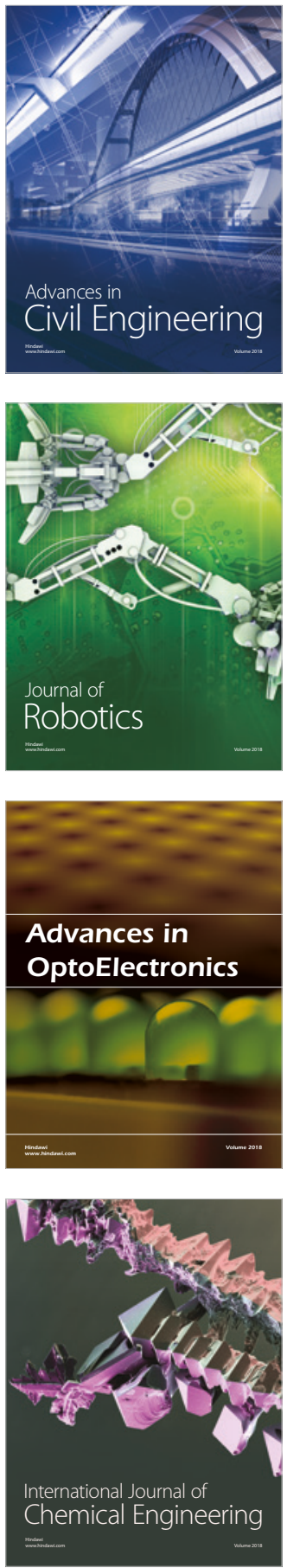

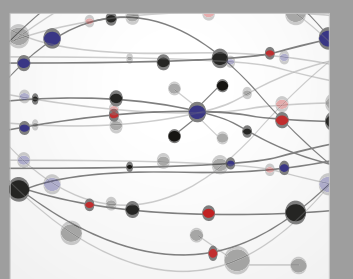

\section{Rotating \\ Machinery}

The Scientific World Journal

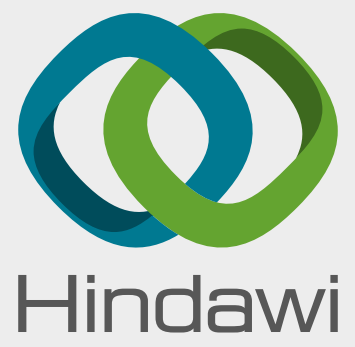

Submit your manuscripts at

www.hindawi.com
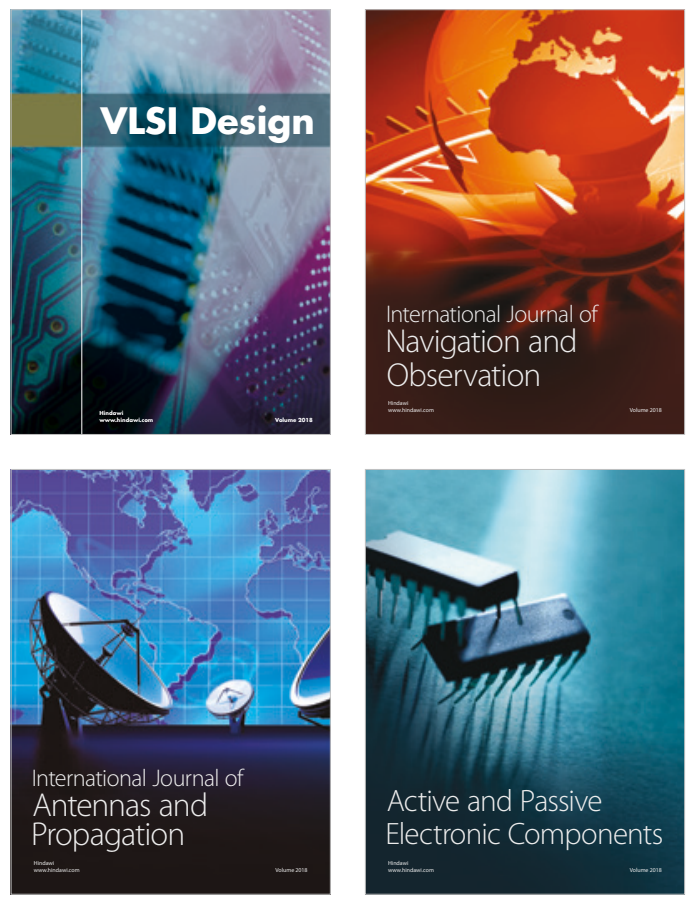
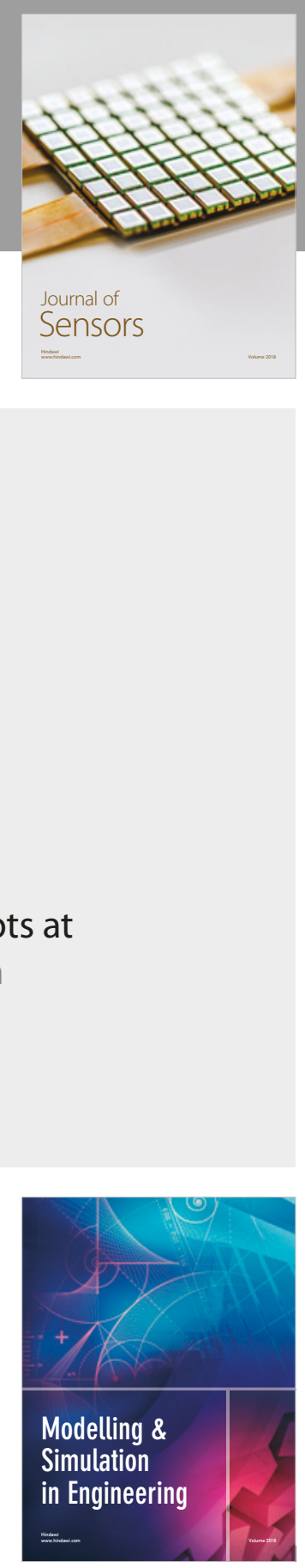

\section{Advances \\ Multimedia}
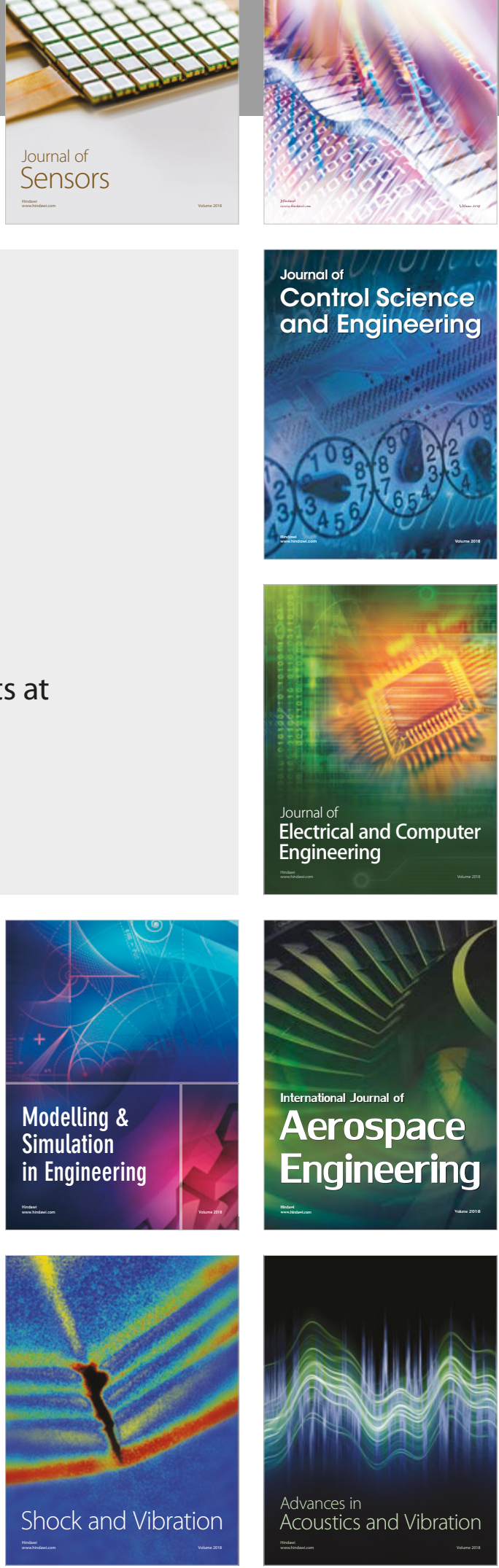